\title{
Immersed boundary method for MHD unsteady natural convection around a hot elliptical cylinder in a cold rhombus enclosure filled with a nanofluid
}

\author{
Ali Akbar Hosseinjani ${ }^{1} \cdot$ Amir H. Roohi ${ }^{1}$
}

Received: 9 September 2020 / Accepted: 14 January 2021 / Published online: 3 February 2021

(c) The Author(s) 2021 OPEN

\begin{abstract}
In this study, the numerical investigation of the natural convection heat transfer around a hot elliptical cylinder inside a cold rhombus enclosure filled with a nanofluid in the presence of a uniform magnetic field is conducted. An immersed boundary method as a computational tool has been extended and applied to solve the problem. The influence of various parameters such as cylinder diameters $(a, b)$, Hartmann number ( $\mathrm{Ha}=0,50$ and 100$)$, nanofluid volume fraction $(\varphi=0,2.5 \%$ and $5 \%)$, and Rayleigh number $\left(\mathrm{Ra}=10^{3}, 10^{4}, 10^{5}, 10^{6}\right.$, and $\left.10^{7}\right)$ has been studied. Streamlines and isotherms contours as well as average Nusselt number have been specified for different modes. An equation for the average Nusselt number as a function of mentioned parameters is presented in this paper. The results show that at lower Ra numbers of $\mathrm{Ra}=10^{3}$ and $10^{4}$, the magnetic field effect is negligible. However, at higher Rayleigh numbers, the average Nusselt number $\left(\mathrm{Nu}_{\mathrm{ave}}\right)$ decreases with the increasing $\mathrm{Ha}$ number. The maximum decrease in $\mathrm{Nu}_{\text {ave }}$ at $\mathrm{Ra}=10^{5}, 10^{6}$ and $10^{7}$ are calculated $-8.15 \%,-23.4 \%$ and $-27.3 \%$, respectively. An asymmetry-unsteady flow is observed at $\mathrm{Ra}=10^{7}$ for $\mathrm{Ha}=0$. However, at higher Ha numbers a steady-symmetrical flow is formed.
\end{abstract}

Keywords Immersed boundary method - MHD · Nanofluid · Natural convection · Average nusselt number · Rhombus enclosure

\begin{tabular}{ll}
\multicolumn{2}{l}{ List of symbols } \\
$\vec{B}$ & Magnetic flux vector \\
$T_{0}$ & Reference temperature $\left({ }^{\circ} \mathrm{K}\right)$ \\
$B_{0}$ & Magnetic flux density \\
$\vec{u}$ & Velocity vector $\left(\mathrm{m} \mathrm{s}^{-1}\right)$ \\
$\overrightarrow{B^{*}}$ & Dimensionless flux vector \\
$\overrightarrow{\mathrm{U}}$ & Dimensionless velocity vector \\
$c_{p}$ & Specific heat at constant pressure $\left(\mathrm{J} \mathrm{kg}^{-1} \mathrm{~K}^{-1}\right)$ \\
$\overrightarrow{\mathrm{U}}^{*}$ & Dimensionless intermediate velocity vector \\
$d_{p}$ & Diameter of the nanoparticle $(\mathrm{m})$ \\
$\vec{V}_{\Gamma}$ & Dimensionless velocity vector on the boundary \\
$d_{f}$ & Diameter of the base fluid molecule $(\mathrm{m})$ \\
$\vec{x}$ & Position vector $(\mathrm{m})$ \\
$d$ & Diameter of cylinder (m)
\end{tabular}

List of symbols

$B \quad$ Magnetic flux vector

$T_{0} \quad$ Reference temperature $\left({ }^{\circ} \mathrm{K}\right)$

$B_{0} \quad$ Magnetic flux density

$\vec{u} \quad$ Velocity vector $\left(\mathrm{m} \mathrm{s}^{-1}\right)$

$B^{*} \quad$ Dimensionless flux vector

Dimensionless velocity vector

$c_{p} \quad$ Specific heat at constant pressure $\left(\mathrm{J} \mathrm{kg}^{-1} \mathrm{~K}^{-1}\right)$

$\vec{U}^{*} \quad$ Dimensionless intermediate velocity vector

$d_{p} \quad$ Diameter of the nanoparticle $(\mathrm{m})$

$\vec{V}_{\Gamma} \quad$ Dimensionless velocity vector on the boundary

$\vec{x} \quad$ Position vector $(\mathrm{m})$

$d \quad$ Diameter of cylinder $(\mathrm{m})$ $\vec{x} \quad$ Dimensionless position vector

D Distribution operator

Greek symbols

$\vec{f} \quad$ Force source to impose velocity boundary condition $\left(\mathrm{m} \mathrm{s}^{-2}\right)$

$\alpha \quad$ Thermal diffusivity $\left(\mathrm{m}^{2} \mathrm{~s}^{-1}\right)$

$\vec{f} \quad$ Dimensionless force source

$\beta \quad$ Coefficient of thermal expansion $\left({ }^{\circ} \mathrm{K}^{-1}\right)$

$\vec{g} \quad$ Gravity acceleration vector $\left(\mathrm{m} \mathrm{s}^{-2}\right)$

$\Theta$ Dimensionless temperature

$h$ Heat source to impose thermal boundary condition

$\Theta^{*}$ Dimensionless intermediate temperature

$\mathrm{H}$ Dimensionless heat source

$\Theta_{\Gamma}$ Dimensionless temperature on the boundary

$\mathrm{Ha}$ Hartmann number

Ali Akbar Hosseinjani, aahosseinjani@qiau.ac.ir; Amir H. Roohi, amir.roohi@qiau.ac.ir| ${ }^{1}$ Department of Mechanical Engineering, Faculty of Industrial and Mechanical Engineering, Qazvin Branch, Islamic Azad University, P. O. Box: 34185-1416, Qazvin, Iran. 
$\gamma \quad$ Angle of the field lines with the positive direction of $x$-axis

I Integration operator

$\mu \quad$ Viscosity $\left(\mathrm{kg} \mathrm{m}^{-1} \mathrm{~s}^{-1}\right)$

$k$ Thermal conductivity $\left(\mathrm{W} \mathrm{m}^{-1} \mathrm{~K}^{-1}\right)$

$\rho \quad$ Density $\left(\mathrm{kg} \mathrm{m}^{-3}\right)$

$\kappa_{b} \quad$ Boltzmann's constant $=1.38066 \mathrm{e}-23\left(\mathrm{JK}^{-1}\right)$

$\phi \quad$ Nanofluid volume fraction

$L_{s} \quad$ Side length of enclosure $(\mathrm{m})$

$\sigma \quad$ Electrical conductivity

$L_{2} \quad L_{2}$ Norm

$\nabla \quad$ Gradient operator

$L_{0} \quad$ Reference length

$\nabla^{2} \quad$ Laplace operator

$M \quad$ Molecular mass of base-fluid

\section{Subscripts}

$N$ Avogadro number

nf Nanofluid

$\mathrm{Nu}$ Local Nusselt number

$f \quad$ Pure fluid

$p \quad$ Pressure (pa)

$p$ Particle

P Dimensionless pressure

fr Freezing point

Pr Prandtl number

H Hot

Re Reynolds number

C Cold

$S$ Distance of cylinders ( $\mathrm{m}$ )

M Momentum

$t$ Time (s)

$\Theta \quad$ Thermal

$T$ Temperature $\left({ }^{\circ} \mathrm{K}\right.$

\section{Introduction}

So far, various engineering applications of natural convection heat transfer inside a nanofluid-filled enclosures are extended. Polymer \& metallurgy industries, hydro-magnetic industries, solar power industries, industrial heat exchangers, food industries, air conditioning systems, heat exchangers in electrical industries, bio systems and medical engineering, and Micro electromechanical systems (MEMS) are examples of the application of nanofluids in heat transfer problems [1-5]. Devendiran and Amirtham [6] have performed a comprehensive review of the application of nanofluids in heat transfer problems. In this review article, they presented a classification of the nanofluid types and its properties as well as the effective thermo-physical properties, including thermal conductivity coefficient, heat capacity, density, and viscosity models for nanofluids. Angayarkanni and Philip [7] reviewed the effective thermal properties of nanofluids and modeling methods of these properties. They have stated that in stable nanofluids, the effective thermal conductivity is independent of temperature. They reported that hybrid nanofluids exhibit higher thermal conductivity than normal nanofluids. Azmi et al. [8] have evaluated the heat transfer coefficients, in particular, based on the determination of the effective thermal conductivity and viscosity. They reported that in all cases, the thermal conductivity of nanofluids is higher, compared to the base fluid. Also, the effective viscosity at low nano-particle volume fraction is independent of the nano-particle type. Kumar Das [9] reviewed the effects of thermal conductivity coefficient in normal and hybrid nanofluids. All the methods, which was reported in various literatures, for modeling the effective thermal conductivity is presented in this review study. Furthermore, they mentioned that the selection of proper hybrid nanoparticles is a major challenge for preparing stable nanofluids. The effective viscosity of nanofluids has been investigated by Murshed and Estellé [10]. Based on the reported results, the nanofluid viscosity is higher than the base fluid in all cases, and the increased nanoparticle volume fraction increases the nanofluid viscosity. Vanaki et al. [1] introduced an extensive overview of the numerical methods used to solve fluid flow problems and heat transfer in nanofluids. They have classified the numerical methods into CFD, single-phase, two-phase, and LBM methods. Also, the presence of a magnetic field could be used to control the heat transfer rate and the fluid streamlines. In this regard, Sheikholeslami and Rokni [11] have reviewed the researches about the effect of magnetic field on heat transfer in nanofluids. The results show that the temperature gradient increases with increasing the nano-particle volume fraction and buoyancy forces, and decreases with the augment of a magnetic field. These widespread applications have led this research area to be investigated in various literatures. The heat transfer area of study in nanofluids under the influence of a magnetic field could be divided into the two following categories:

The first category is the investigation of heat transfer problems in an enclosure. Sarris et al. [12] studied the MHD convective heat transfer in a square enclosure with a cold wall and a laterally air-filled hot wall. They reported that in high Rayleigh numbers an unsteady flow is formed, and an increase in the Hartmann number at some levels of Rayleigh numbers results in reduced or vanished flow oscillations. Oztop et al. [13] investigated MHD convective heat transfer in a lid-driven cavity with a corner heater. They showed that the rate of heat transfer decreases with an increase in the Hartmann number, and this decreased rate of heat transfer is more significant at higher Grashof numbers. Kefayati [14] determined the natural convection 
under the influence of a magnetic field in a $\mathrm{Cu} /$ Water filled enclosure by LBM approach. It was concluded that the effect of nano-particle concentration on the rate of heat transfer varies in different Rayleigh numbers: In $R a=10^{3}$, the effect of nano-particles on the heat transfer rate decreases with the increasing Hartmann number. While, at higher Rayleigh numbers (i.e., $\mathrm{Ra}=10^{5}$ ) this effect increases with the increasing Hartmann number. Magneto hydrodynamic natural convection in an open enclosure filled with Cu-water nanofluid is studied numerically by Hussein et al. via LBM [15]. They reported that the solid volume fraction has a significant influence on stream function and heat transfer, depending on the value of $\mathrm{Ha}$ and Ra numbers. Mahmoudi et al. [16] studied the effect of magnetic field on the rate of heat transfer in a triangular enclosure filled with nanofluids. They reported that the maximum decrease in the average Nusselt number occurs with an increase in the Hartmann number at $\mathrm{Ra}=10^{6}$. In another study, Oztop et al. [17] investigated the MHD natural convection in an enclosure filled with a nanofluid from two semi-circular heaters on the bottom wall. They observed that the Hartmann number has an inverse effect on the rate of heat transfer, and the distance between the two heaters would have an important influence on the streamline and heat transfer values. MHD natural convection inside a T-shape enclosure filled with nanofluid has been investigated numerically by Hussein et al. [18]. The results show that the Nu number increases with the increase of Ra number, inclination angle, aspect ratio, heat source location and nanoparticles and it decreases when the Ha number and heat source length increase. Ghasemi [19] specified the natural heat transfer in a $U$-shaped enclosure filled with nanofluids under the influence of a magnetic field. Results show that the heat transfer rate increases with an increase in Rayleigh number and volume fraction of nanofluid, and decreases as Hartmann number increases. Sheremet et al. [20] conducted a numerical investigation of the natural convection heat transfer in an inclined wavy enclosure filled with nanofluid, when a uniform magnetic field was also present. Results showed that the variations of inclination angle would result in significant changes of the fluid flow and heat transfer inside the enclosure. Numerical modeling of the natural convection heat transfer inside a nanofluid filled triangular enclosure with a semi-circular bottom wall was carried out by Dogonchi et al. [21]. They used a single-phase model for nanofluid modeling, and proposed a mathematical model for the determination of the average Nusselt number. Al-Rashed et al. [22] performed a numerical flow analysis of a 3D CNTnanofluid filled enclosure under a uniform active magnetic field. Results showed that the location of magnetic field has a significant effect on the rate of heat transfer, even if the Hartmann number is constant. Hajatzadeh et al.
[23] carried out the numerical study of the natural convection heat transfer and entropy generation inside an enclosure filled with Al2O3/water mixture. A uniform and non-uniform boundary conditions have been investigated in several cases as well as different Hartmann numbers. The results showed that with an increasing nano-particle volume fraction, Nusselt number and entropy generation increase in all cases. Alsabery et al. [24] investigated the forced convection heat transfer inside a lid-driven enclosure with a heated triangular wall. In this regard, they used a two-phase nanofluid model. The results show that increasing the Hartmann number leads to an increased Brownian motion, which increases the migration of the nano-particles. Selimefendigile and Oztop [25] investigated the effect of conductive wall on MHD natural convection in a CNT/water filled enclosure. They observed that the average heat transfer increases with an increase in the Rayleigh number and the nano-particles volume fraction. Furthermore, the maximum Nusselt number of $84 \%$ is achieved when the volume fraction of $3 \%$ is present. Similarly, an increase in the Hartmann number reduces the average Nusselt number. A 3D numerical modeling of MHD convection heat transfer inside a cubical cavity filled with CNT-water nanofluid has been done by Al-Rashed et al.[26] they reported that the heat transfer rate intensify with increasing CNT particle and Ra number and decrease by $50 \%$ when $\mathrm{Ha}$ increase from 50 to 100 .

The second category is the investigation of heat transfer problems in an enclosure, when there is an obstacle inside it. Sheikholeslami et al. [27] utilized LBM to study the heat transfer problem of an elliptic cylinder in a square enclosure filled with nanofluids. The effective thermal conductivity and viscosity of nanofluids were calculated using KKL (Koo-Kleinstreuer-Li) correlation. Similarly, the results show that the average Nusselt number decreases with increasing Hartmann number, and increases with increasing Rayleigh number and nanofluid volume fraction. In addition, the effect of Hartmann number would be less on higher Rayleigh numbers. Ashorynejad et al. [28] studied the effect of a magnetic field on the natural convection of nanofluids in a horizontal cylindrical annulus enclosures. By using the LBM method in their numerical modeling, results showed that average Nusselt number increases with increasing nano-particle volume fraction and Rayleigh number. Selimefendigile and Oztop [29] investigated MHD mixed convection in a triangular enclosure filled with nanofluids with an inner rotating circular adiabatic cylinder. They have reported that the rate of heat transfer increases with an increase in the Grashof number and nano-particle volume fraction as well as the cylinder rotational speed. In another study, Selimefendigile and Oztop [30] investigated the heat transfer and entropy generation problem in an enclosure with different shaped obstacles, 
including square, triangular and diamond shaped geometries filled by nanofluids. They have concluded that the presence of an obstacle inside the enclosure leads to a decreased rate of heat transfer, compared to the cases without an obstacle. Hassanpour et al. [31] conducted the numerical study of the forced convection heat transfer in a nanofluid filled square enclosure with an inner circular cylinder under a magnetic field and a constant heat flux. They have reported that an increase in Reynolds and Hartmann numbers would lead to an increase in the average Nusselt number. Kefayati and Tang [32] performed the numerical analysis of MHD natural convection and entropy generation in a non-Newtonian fluid filled enclosure with two circular cylinders. As stated, the entropy generation decreases with increasing Hartmann number. Numerical study of forced convection heat transfer and entropy generation in a lid-driven enclosure with two rotating cylinders filled with a nanofluid in the presence of a magnetic field is done by Barnoon et al. [33]. They have utilized a two-phase model to simulate the flow and heat transfer in nanofluids. The results showed that with a decrease in Hartmann number and Richardson number as well as an increase in the nano-particle volume fraction, the rate of heat transfer would increase. Daneshvar et al. [34] modeled MHD forced convection in an enclosure with two rotating cylinders in different configurations. In this regard, a two-phase model was used to analyse the forced convection heat transfer in a nanofluid. They concluded that an increase in the Hartmann number results in a decrease in heat transfer rate, and the maximum effect of magnetic field appears in the vertically alignment of two rotating cylinders. Alnaqi et al. [35] investigated the natural convection heat transfer and entropy generation in a nanofluid filled diagonal square enclosure equipped with a conductor fin. The radiation effects and magnetic field were considered in this study. According to the results, the rate of heat transfer increases by increasing the radiation parameter with respect to higher Rayleigh numbers. Alkanhal et al. [36] performed a numerical analysis of the effect of Lorentz force on the nanofluid behavior in a permeable enclosure filled with nanofluids in porous media; where an elliptic obstacle was under the influence of a magnetic field. The results showed that entropy generation would increase with an increase in Hartmann number. Tayebi and Chamkha [37] studied the heat transfer and entropy generation in a Cu-Al2O3/water filled enclosure. Inside this enclosurea hollow conductive cylinder was placed. They extended Corcione correlation in order to calculate the effective thermal conductivity and viscosity. As well, Tayebi and Chamkha [38] investigated the heat transfer of a nanofluid filled enclosure with a wavy circular conductive cylinder under the influence of a magnetic field. Based on the results, the geometrical characteristics of the wavy cylinder have an important role in the rate of heat transfer and the flow direction. Vijaybabu and Dhinakaran [39] studied the heat transfer inside a square enclosure with a permeable triangular cylinder as a function of different geometrical parameters using the LBM method. The enclosure was filled with Al2O3/water nanofluid and affected by a magnetic field. The results show that an increase in Hartmann number would lead to a decrease in permeability and intensity of heat transfer.

In this manuscript, a numerical analysis based on the iterative direct forcing immersed boundary method is developed for the determination of streamlines and heat transfer in a rhombus enclosure with an inner elliptical cylinder filled with $\mathrm{Cu} /$ water nanofluid. The flow field is influenced by an external magnetic field that is applied horizontally. Actually, at the present paper, the effects of magnetic field on the natural convection heat transfer in nanofluid have been numerically modeled and analysis which isn't studied at previous references such as [40].

So far, the use of the IBM method is not reported in the literature in order to solve the MHD natural convection problems. In this study, a statistical equation is also proposed for the average Nusselt number as a function of input parameters (i.e., cylinder geometrical parameter, Hartmann number, nanofluid volume fraction, and Rayleigh number). An asymmetry-unsteady flow is observed at Rayleigh number of $\mathrm{Ra}=10^{7}$ and Hartmann number of $\mathrm{Ha}=0$ (i.e., when no magnetic field is applied). Also, with Hartman numbers of $\mathrm{Ha}=50$ and $\mathrm{Ha}=100$, the flow field would be steady.

The rest of this paper is arranged as follows: Governing equations, numerical method description, problem definition, numerical method validation, and results and discussion.

\section{Governing equations and nanofluid modeling}

The governing equations for an incompressible transient laminar flow with natural heat convection and an imposed magnetic field in the computational domain are as follows [11] and [40]:

$\frac{\partial \vec{u}}{\partial t}+\vec{u} . \nabla \vec{u}=-\frac{1}{\rho_{n f}} \nabla p+\frac{\mu_{n f}}{\rho_{n f}} \nabla^{2} \vec{u}+\frac{\sigma_{n f}}{\rho_{n f}}(\vec{u} \times \vec{B}) \times \vec{B}+\frac{(\rho \beta)_{n f}}{\rho_{n f}} \vec{g}\left(T-T_{0}\right)+\vec{f}$ 
$\frac{\partial T}{\partial t}+\vec{u} . \nabla T=\alpha_{n f} \nabla^{2} T+h$

$\nabla \cdot \vec{u}=0$.

In the above equations, $\vec{f}$ is the external momentum source used to impose the no-slip boundary condition in IBM, $h$ is the external heat source, which is added to the energy equation in order to implement the specified thermal boundary condition in IBM (more details on implementing immersed boundary method would be found in $[41,42]$ and [40]). The third and fourth term, respectively, on the right hand side of Eq. (1) is the magnetic field effect and the Boussinesq approximation. Note that the former stands for thermal natural convection effect. $\rho_{n f}$ is the effective nanofluid density, $\mu_{n f}$ is the effective nanofluid viscosity, $\vec{u}=u \hat{i}+v \hat{j}$ is the velocity vector, and $\vec{B}=B_{o} \cos \gamma \hat{i}+B_{o} \sin \gamma \hat{j}$ is the vector of uniform magnetic field. It is worth mentioning that $B_{0}$ is the magnitude of the magnetic field, and $\gamma$ is the angle of the field lines with the positive direction of the $x$-axis (i.e., in the present study $\gamma=0$ ). As well, $p$ is the pressure, $\vec{g}$ is the gravitational acceleration vector, $a_{n f}=k_{n f} /\left(\rho c_{p}\right)_{n f}$ is the effective thermal diffusivity. Finally, $\beta_{n f}$ is the effective thermal expansion coefficient as follows:

$\beta_{n f}=\beta_{f}(1-\varphi)+\beta_{s} \varphi$

where, $\beta_{s}$ is the thermal expansion coefficient of nanoparticles, and $\beta_{f}$ is the thermal expansion coefficient of the base-fluid.

The effective nanofluid viscosity is defined as Eq. (5), proposed by Corcione [43] as:

$\frac{\mu_{n f}}{\mu_{f}}=\frac{1}{1-34.87\left(d_{p} / d_{f}\right)^{-0.3} \varphi^{1.03}}$

where, $d_{f}$ is the equivalent diameter of the base-fluid molecule, which is calculated from the following equation:

$d_{f}=0.1\left(\frac{6 M}{N \pi \rho_{f 0}}\right)^{1 / 3}$

$\frac{\partial \vec{U}}{\partial t}+\vec{U} \cdot \nabla \vec{U}=-\nabla P+\operatorname{Pr} \frac{A_{5} A_{2}}{A_{1} A_{4}} \nabla^{2} \vec{U}+H^{2} \operatorname{Pr} \frac{A_{6} A_{2}}{A_{1} A_{4}}\left(\vec{U} \times \overrightarrow{B^{*}}\right) \times \overrightarrow{B^{*}}+\operatorname{RaPr} \frac{A_{3} A_{2}^{2}}{A_{1} A_{4}^{2}} \Theta+\vec{f}$
In the above equation, $M$ is the molecular mass of basefluid, $\mathrm{N}$ is Avogadro number, and $\rho_{\mathrm{fo}}$ is the base-fluid density at the standard temperature of $\mathrm{T}_{0}=293^{\circ} \mathrm{K}$.

On the other hand, the effective thermal conductivity coefficient of the nanofluid is also proposed by Corcione as follows:

$\frac{k_{n f}}{k_{f}}=1+4.4 \operatorname{Re}^{0.4} \operatorname{Pr}^{0.66}\left(\frac{T}{T_{f r}}\right)^{10}\left(\frac{k_{p}}{k_{f}}\right)^{0.03} \varphi^{0.66}$

where, Re is the Reynolds number of the nano-particles, and $\operatorname{Pr}=\mu_{f} / \rho_{f} \alpha_{f}$ is the Prandtl number of the base-fluid. Also, $T_{f r}$ is the freezing point of the base-fluid. The nanoparticle Reynolds number is defined as Eq. (8):

$\operatorname{Re}=\frac{2 \rho_{f} \kappa_{b} T}{\pi \mu_{f}^{2} d_{p}}$

In Eq. (8), $K_{b}$ is the Boltzmann constant, $\sigma_{n f}$ is the effective electrical conductivity of the nanofluid, which is defined as follows [44]:

$\frac{\sigma_{n f}}{\sigma_{f}}=1+\frac{3\left(\sigma_{s / \sigma_{f}}-1\right) \varphi}{\left(\sigma_{s} / \sigma_{f}+2\right)-\left(\sigma_{s} / \sigma_{f}-1\right) \varphi}$

Other effective thermo-physical properties of the nanofluids are calculated by following equations:

$(\rho \beta)_{n f}=(\rho \beta)_{f}(1-\varphi)+(\rho \beta)_{s} \varphi$

$\rho_{n f}=\rho_{f}(1-\varphi)+\rho_{s} \varphi$

$\left(\rho c_{p}\right)_{n f}=\left(\rho c_{p}\right)_{f}(1-\varphi)+\left(\rho c_{p}\right)_{s} \varphi$

The dimensionless form of mentioned equations is as follows:

$\overrightarrow{\mathrm{U}}=\frac{\vec{u} L_{0}}{\alpha_{f}}, \overrightarrow{\mathrm{X}}=\frac{\vec{x}}{L_{0}}, \mathrm{t}=\frac{t \alpha_{f}}{L_{0}^{2}}, \mathrm{P}=\frac{p L_{0}^{2}}{\rho_{n f} \alpha_{f}^{2}}, \Theta=\frac{T-T_{C}}{T_{H}-T_{C}}, \overrightarrow{\mathrm{B}^{*}}=\frac{\vec{B}}{B_{0}}$

where, $L_{0}$ is the reference length that is proportional to the side length of the enclosure, and $T_{H}$ and $T_{C}$ are the cold and hot source temperatures at the body boundaries, respectively. $a_{f}$ is the thermal diffusivity of the base-fluid.

By replacing these values in Eqs. (1-3) the dimensionless form of the governing equations obtains as follows: 
$\frac{\partial \Theta}{\partial \mathrm{t}}+\overrightarrow{\mathrm{U}} \cdot \nabla \Theta=\frac{\mathrm{A}_{4}}{\mathrm{~A}_{2}} \nabla^{2} \Theta+\mathrm{h}$

In the above equations, the constant coefficients are defined as follows:

$\mathrm{A}_{1}=\frac{\rho_{n f}}{\rho_{f}}, \mathrm{~A}_{2}=\frac{\left(\rho C_{p}\right)_{n f}}{\left(\rho C_{p}\right)_{f}}, \mathrm{~A}_{3}=\frac{(\rho \beta)_{n f}}{(\rho \beta)_{f}}, \mathrm{~A}_{4}=\frac{k_{n f}}{k_{f}}, \mathrm{~A}_{5}=\frac{\mu_{n f}}{\mu_{f}}, \mathrm{~A}_{6}=\frac{\sigma_{n f}}{\sigma_{f}}$

The terms of the force and the heat source used in the iterative direct forcing immersed boundary method for applying boundary conditions would be dimensionless as Eqs. (17) and (18):

$\overrightarrow{\mathrm{f}}=\frac{\vec{f} L_{0}^{3}}{\alpha_{f}^{2}}$

$\mathrm{h}=\frac{h L_{0}^{2}}{\alpha_{f}\left(T_{H}-T_{C}\right)}$

Also, the dimensionless numbers in the dimensionless governing equations (Eq. (14) and Eq. (15)) are as follows:

$\mathrm{Ra}=\frac{\rho_{f} \vec{g} \beta_{f}\left(T_{H}-T_{C}\right) L_{0}^{3}}{\mu_{f} \alpha_{f}}$

$\mathrm{Ha}=L_{0} B_{0} \sqrt{\frac{\sigma_{f}}{\mu_{f}}}$

The constant temperature and no-slip boundary conditions on the cylinder walls are:

$\vec{U}_{B}=\vec{V}_{\Gamma}$

$\Theta_{\mathrm{B}}=\Theta_{\Gamma}$

Local and average Nusselt numbers are also defined, respectively, as follows:

$\mathrm{Nu}=-\frac{k_{n f}}{k_{f}}\left(\frac{\partial \Theta}{\partial \overrightarrow{\mathrm{X}}}\right)_{\text {wall }}$
$\mathrm{Nu}_{\mathrm{ave}}=\frac{1}{S} \int_{0}^{S} \mathrm{Nuds}$

In the current problem, water is used as base fluid and copper as nano-particle. The thermo-physical properties of the materials are listed in Table 1.

\section{Numerical method}

In this study, the immersed boundary method (IBM) is used to solve the governing equations. In fact, the IBM method presented in references [42] and [40] is developed in such a way to solve the natural convection heat transfer problems in nanofluids and considering the presence of a magnetic field, as well. Hence, the developed method is applied to study the effect of magnetic field on the flow control and the heat transfer.

\subsection{Immersed boundary method}

The Immersed boundary method was first used by Peskin [45] to model the blood flow in the heart Cardiac valve. The main advantage of this method was that it used a uniform Cartesian grid, which did not match the exact shape of the heart. Despite the use of Cartesian grid, it well recognized the existence of boundaries. So far, there have been many revisions to this method. In addition, some other methods such as the direct forcing method, and cut cell method have also been developed in the area of IBM method [42]. According to Fig. 1, the solid part $\left(\Omega_{\mathrm{b}}\right)$ is surrounded by the fluid region $\left(\Omega_{\mathrm{f}}\right)$. The boundary is also marked by $\Gamma_{\mathrm{b}}$. In the immersed boundary method, the governing equations are discretized over a uniform grid regardless of the object boundary. The boundary effects (i.e., boundary conditions) are applied indirectly by the source terms. There are two types of nodes here. The boundary nodes, denoted by $\vec{X}$ in Fig. 1a, are called the Lagrangian nodes, in which the source terms are calculated. The grid nodes, denoted by $\vec{x}$ in Fig. $1 \mathrm{a}$, are called Eulerian nodes. The source terms are calculated on the Lagrangian nodes, which then are distributed over the Eulerian nodes by a distribution function. Finally, the governing equations are solved with consideration of these terms. In this method, the boundary conditions are applied indirectly by these terms to
Table 1 Thermo-physical properties of water and nanoparticles at $\mathrm{T}=320[40]$

\begin{tabular}{llllllll}
\hline Property & $\rho$ & $\mathrm{K}$ & $\mathrm{C}_{\mathrm{p}}$ & $\beta \times 10^{-5}$ & $\mu \times 10^{-6}$ & $\mathrm{~d}_{\mathrm{p}}$ & $\sigma$ \\
\hline Unit & {$\left[\mathrm{kg} / \mathrm{m}^{3}\right]$} & {$[\mathrm{M} / \mathrm{mK}]$} & {$[\mathrm{J} / \mathrm{kgK}]$} & {$\left[\mathrm{K}^{-1}\right]$} & {$\left[\mathrm{kgm}^{-1} \mathrm{~s}^{-1}\right]$} & {$[\mathrm{nm}]$} & {$\left[\Omega \mathrm{m}^{-1}\right.$} \\
Cu & 8933 & 401 & 385 & 1.67 & - & 45 & $5.96 \times 10^{7}$ \\
Water & 997.1 & 0.613 & 4179 & 21.2 & 695 & - & 0.05 \\
\hline
\end{tabular}



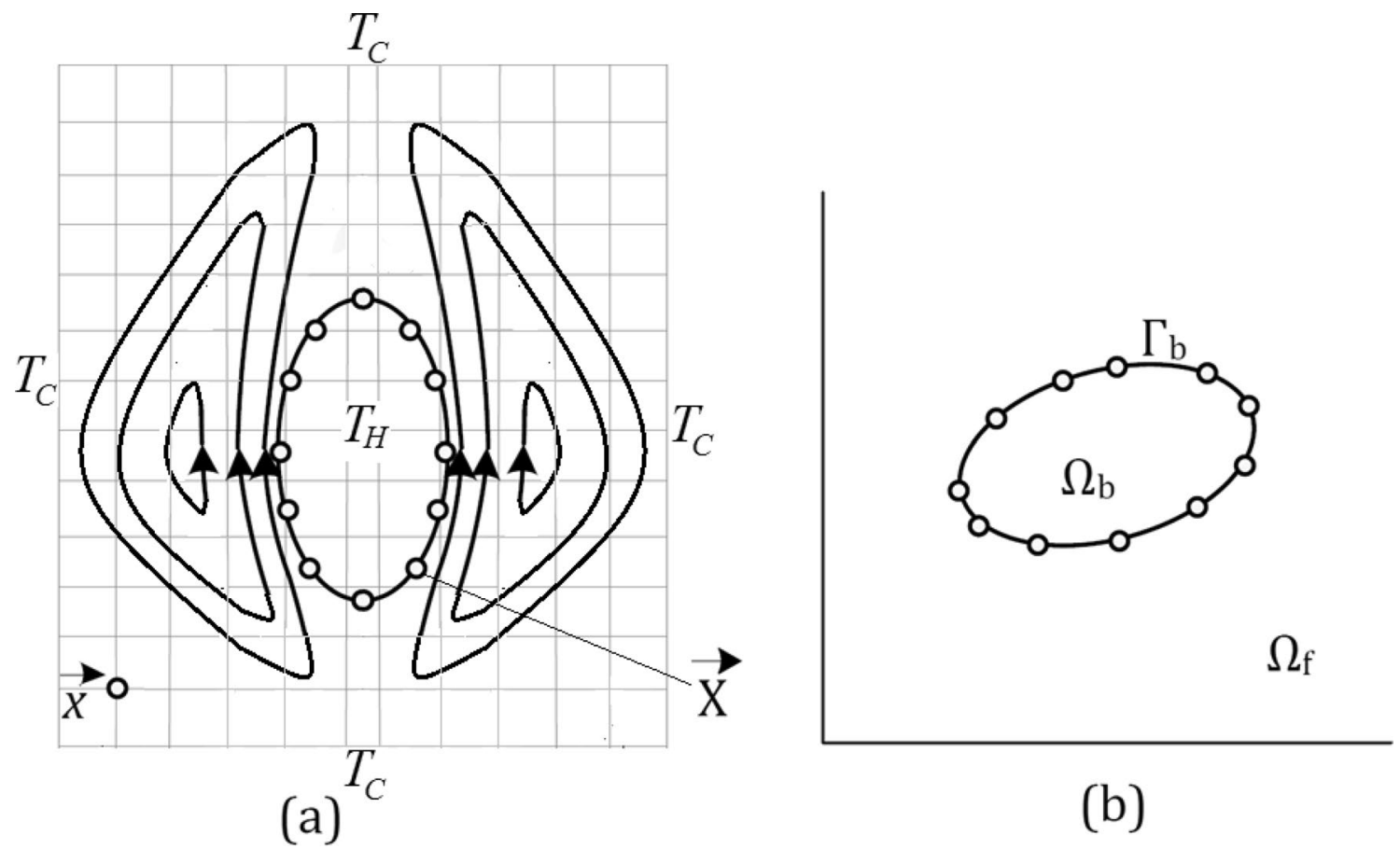

Fig. 1 Computational field and grid points (Eulerian); and boundary points (Lagrangian)

the governing equations. It is worth mentioning that the method is effective at complex and moving boundaries, in particular.

\subsection{Iterative direct forcing IBM}

In this study, an IBM method presented in literatures [40, 42] and [46] is used to solve the problem of fluid flow right hand side of Eq. (15) is used in order to apply the temperature boundary condition. In fact, the purpose is to calculate these source terms, according to the boundary conditions and the governing equations in a way that the boundary conditions could be applied indirectly to the Lagrangian nodes. Iterative direct forcing IBM uses the governing equations to compute these source terms. To achieve this, separating Eqs. (14) and (15) results in:

$\vec{U}^{n+1}=\vec{U}^{n}+\delta t\left(\frac{3}{2} h_{m}^{n}-\frac{1}{2} h_{m}^{n-1}-\frac{3}{2} \nabla P^{n+1}+\frac{1}{2} \nabla P^{n}+\frac{3}{2} h_{e}^{n}-\frac{1}{2} h_{e}^{n-1}+\frac{3}{2} \frac{A_{3} A_{2}^{2}}{A_{1} A_{4}^{2}} \operatorname{RaPr} \Theta^{n+1}-\frac{1}{2} \frac{A_{3} A_{2}^{2}}{A_{1} A_{4}^{2}} \operatorname{Ra} \operatorname{Pr} \Theta^{n}\right)+\vec{f}^{n+\frac{1}{2}} \delta t$

and heat transfer in nanofluids in the presence of a magnetic field. As is described in [46], two distribution $D(\Phi)$ and integration $\mathrm{I}(\varnothing)$ operators are used to calculate the force within the boundaries of the flow field. $\varnothing$ Parameter substitutes physical and thermal parameters such as $\vec{U}, \vec{f}, \Theta$, and $h$ at the Eulerian nodes. As well, $\Phi$ parameter substitutes the defined parameters such as $\vec{V}_{\Gamma}, \vec{F}, \Theta_{\Gamma}$, and $\mathrm{H}$ on boundary points (i.e., Lagrangian nodes). Recall that in IBM, boundary conditions are applied by source terms. The force source term, $\vec{f}$, in the right side of the momentum equation (Eq. (14)) is used in order to apply the velocity boundary condition. Also, the heat source term, $h$, in the
$\Theta^{n+1}=\Theta^{n}+\delta t\left(\frac{3}{2} h_{\Theta}^{n}-\frac{1}{2} h_{\Theta}^{n-1}\right)+h^{n+\frac{1}{2}} \delta t$.

where, $h_{m}$ is equal to $h_{m}=-\vec{U} . \nabla \vec{U}+\operatorname{Pr} \frac{A_{5} A_{2}}{A_{1} A_{4}} \nabla^{2} \vec{U}$, which contains the convective and diffusive terms of the momentum equation. As well, $h_{e}=\mathrm{Ha}^{2} \operatorname{Pr} \frac{A_{6} A_{2}}{A_{1} A_{4}}\left(\vec{U} \times \overrightarrow{B^{*}}\right) \times \overrightarrow{B^{*}}$ considers the effect of the Lorentz force term on the governing equation. Obviously, these equations are written in the vector form; the Lorentz force term in the two-dimensional component form is as follows: 
$\left(\vec{U} \times \overrightarrow{B^{*}}\right) \times \overrightarrow{B^{*}}=\left|B^{*}\right|^{2}\left(v^{*} \sin \lambda \cos \lambda-u^{*} \sin ^{2} \lambda\right) \hat{i}+\left|B^{*}\right|^{2}\left(u^{*} \sin \lambda \cos \lambda-v^{*} \cos ^{2} \lambda\right) \hat{j}$

In addition, $\mathrm{h}_{\Theta}=-\overrightarrow{\mathrm{U}} . \nabla \Theta+\frac{\mathrm{A}_{4}}{\mathrm{~A}_{2}} \nabla^{2} \Theta$ includes the convective and diffusive terms in the energy equation. Note that $\mathrm{h}_{\mathrm{m}}, \mathrm{h}_{\mathrm{e}}$, and $\mathrm{h}_{\Theta}$ terms as well as the pressure term are separated by the second-order Adams-Bashforth method. All position and temperature derivatives are separated by the central second-order method. The numerical stability is achieved by transit solution of the governing equations. The velocity and temperature boundary condition at Lagrangian nodes is defined as follows; based on the integration operator previously mentioned:
$I\left(\vec{f}^{n+\frac{1}{2}} \delta t\right)=\vec{V}_{\Gamma}-1\left(\vec{U}^{*}-\frac{3}{2} \nabla P^{n+1}+\frac{3}{2} \frac{A_{3} A_{2}^{2}}{A_{1} A_{4}^{2}} \operatorname{Ra} \operatorname{Pr} \Theta^{n+1}\right)$

$\mathrm{I}\left(\mathrm{h}^{\mathrm{n}+\frac{1}{2}} \delta t\right)=\Theta_{\Gamma}-\mathrm{I}\left(\Theta^{*}\right)$

Using the distribution operator $D$, the force source and heat source terms (i.e. $\vec{f}^{n+\frac{1}{2}}$ and $h^{n+\frac{1}{2}}$ ) at the Eulerian nodes (i.e., grid points) for applying boundary conditions are as follows:

$\vec{f}^{n+\frac{1}{2}} \delta t=D\left(I\left(\vec{f}^{n+\frac{1}{2}} \delta t\right)\right)=D\left(\vec{V}_{\Gamma}-1\left(\vec{U}^{*}-\frac{3}{2} \nabla P^{n+1}+\frac{3}{2} \frac{A_{3} A_{2}^{2}}{A_{1} A_{4}^{2}} \operatorname{Ra} \operatorname{Pr} \Theta^{n+1}\right)\right)$

$\mathrm{I}\left(\overrightarrow{\mathrm{U}}^{\mathrm{n}+1}\right)=\overrightarrow{\mathrm{V}}_{\Gamma}$

$\mathrm{I}\left(\Theta^{\mathrm{n}+1}\right)=\Theta_{\Gamma}$

In Eqs. (28) and (29), I $\left(\vec{U}^{n+1}\right)$ and $\left(\Theta^{n+1}\right)$ are dimensionless velocity and temperature values at Lagrangian nodes on the inner boundaries, respectively.

$\vec{U}^{*}$ and $\Theta^{*}$ are the intermediate velocity and temperature values, respectively, defined as Eqs. (30) and (31). This intermediate velocity and temperature values would be used in the numerical solution process.

$\vec{U}^{*}=\vec{U}^{n}+\delta t\left(\frac{3}{2} h_{m}^{n}-\frac{1}{2} h_{m}^{n-1}+\frac{1}{2} \nabla P^{n}+\frac{3}{2} h_{e}^{n}-\frac{1}{2} h_{e}^{n-1}-\frac{1}{2} \frac{A_{3} A_{2}^{2}}{A_{1} A_{4}^{2}} R a \operatorname{Pr} \Theta^{n}\right)$

$\Theta^{*}=\Theta^{n}+\delta t\left(\frac{3}{2} h_{\Theta}^{n}-\frac{1}{2} h_{\Theta}^{n-1}\right)$

By substituting Eqs. (30) and (31) into the Eqs. (25) and (26), and considering the Eqs. (28) and (29):

$\vec{V}_{\Gamma}=1\left(\vec{U}^{n+1}\right)=1\left(\vec{U}^{*}-\frac{3}{2} \nabla P^{n+1}+\frac{3}{2} \frac{A_{3} A_{2}^{2}}{A_{1} A_{4}^{2}} \operatorname{RaPr} \Theta^{n+1}\right)+1\left(\vec{f}^{n+\frac{1}{2}} \delta t\right)$

$\Theta_{\Gamma}=\mathrm{I}\left(\Theta^{\mathrm{n}+1}\right)=\mathrm{I}\left(\Theta^{*}\right)+\mathrm{I}\left(\mathrm{h}^{\mathrm{n}+\frac{1}{2}} \delta t\right)$

The above equations could be rewritten as follows: $\mathrm{h}^{\mathrm{n}+\frac{1}{2}} \delta t=\mathrm{D}\left(\mathrm{I}\left(\mathrm{h}^{\mathrm{n}+\frac{1}{2}} \delta t\right)\right)=\mathrm{D}\left(\Theta_{\Gamma}-\mathrm{I}\left(\Theta^{*}\right)\right)$

These source terms, derived directly from the governing equation and boundary conditions, would be applied to the Cartesian grid nodes (Eulerian points) in order to apply the velocity and temperature boundary conditions. Other details of the current algorithm are the same as the described approaches in literatures [42] and [40].

\section{Problem definition}

The boundary conditions and the two-dimensional solution field are shown in Fig. 2. The magnetic field affects the solution field horizontally. Here, $L$ is the reference length

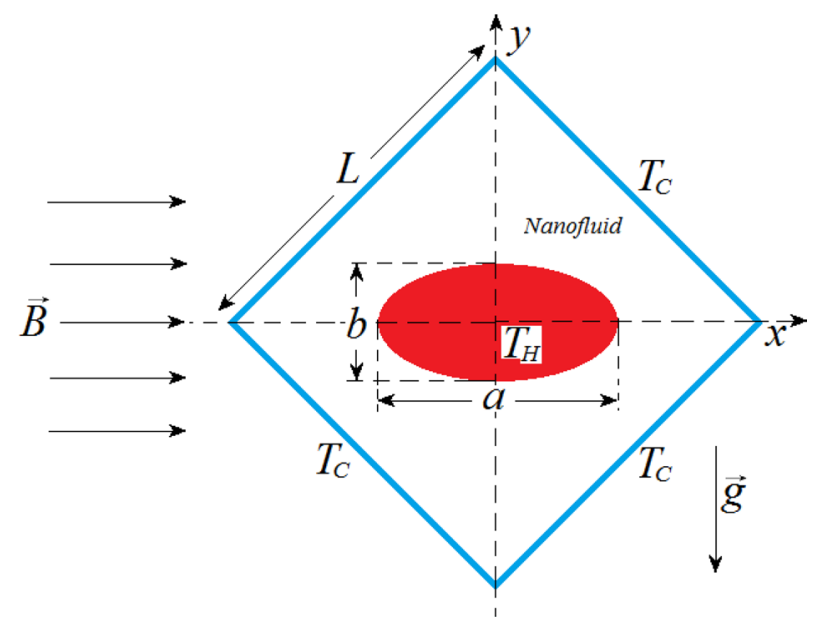

Fig. 2 Boundary conditions and the two-dimensional solution field of the problem 


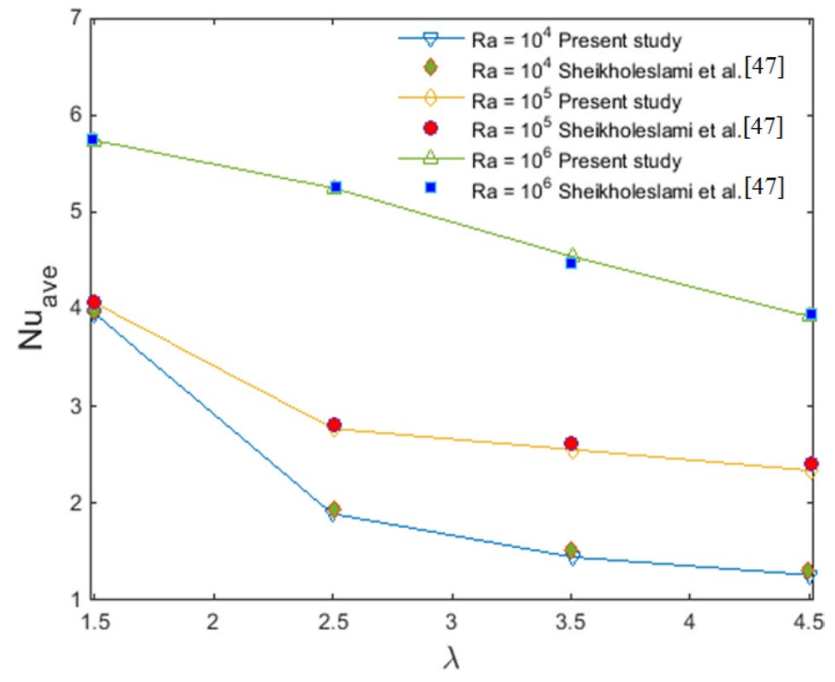

Fig. $3 \mathrm{Nu}_{\text {ave }}$ along the surface of cold wall

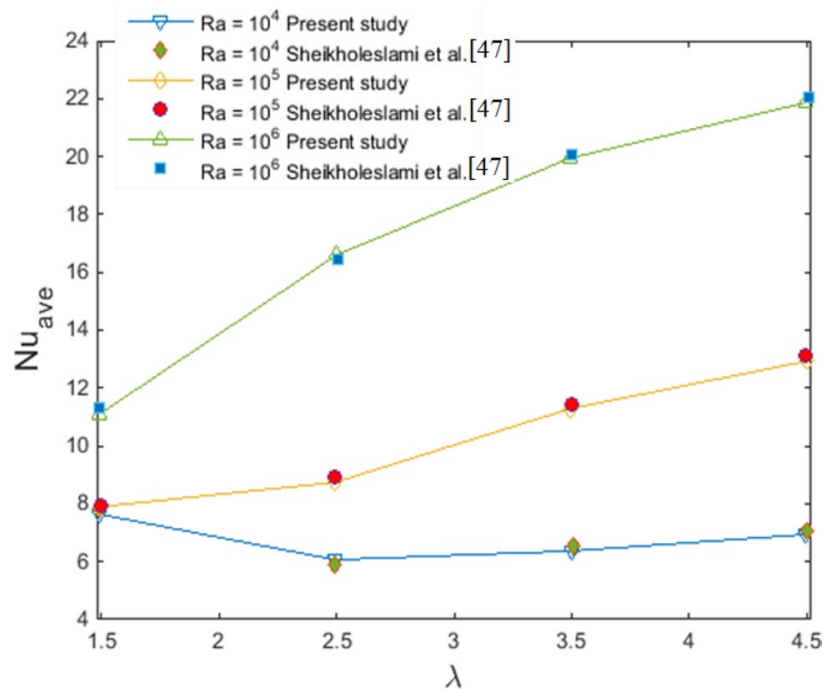

Fig. $4 \mathrm{Nu}_{\text {ave }}$ along the surface of hot cylinder

and two geometrical parameters $a$ and $b$ are sketched with respect to this length. The hot elliptical cylinder is placed inside a cold enclosure. The elliptical cylinder is assumed to be fixed, and a natural convection heat transfer occurs. The shape parameters are considered in the range of $0.2 \mathrm{~L} \leq \mathrm{a} \leq 0.6 \mathrm{~L}$ and $0.2 \mathrm{~L} \leq \mathrm{b} \leq 0.6 \mathrm{~L}$ for a nano-particle with a volume fraction of $0 \leq \varphi \leq 5 \%$, and Hartmann number of $0 \leq \mathrm{Ha} \leq 100$. Also, the problem is solved for the Rayleigh numbers of $R a=10^{3}, 10^{4}, 10^{5}, 10^{6}$, and $10^{7}$. A view of utilized mesh has been shown in "Appendix".
Table 2 Grid independence evaluation of this study

\begin{tabular}{lccccc}
\hline Mesh size & $170 \times 170$ & $190 \times 190$ & $200 \times 200$ & $210 \times 210$ & $220 \times 220$ \\
\hline $\begin{array}{l}N u_{\text {ave }} \\
\text { (along }\end{array}$ & 4.126 & 4.116 & 4.095 & 4.083 & 4.082 \\
the \\
$\begin{array}{l}\text { surface } \\
\text { of cold } \\
\text { wall) }\end{array}$ & & & & & \\
$\begin{array}{l}\text { Nu } \\
\text { ave }\end{array}$ & 21.761 & 21.905 & 22.003 & 22.017 & 22.019 \\
$\begin{array}{l}\text { (along } \\
\text { the } \\
\text { surface } \\
\text { of hot } \\
\text { cylinder) }\end{array}$ & & & & & \\
\hline
\end{tabular}

\section{Validation study}

The numerical method had been evaluated for natural convection problems modeling in [40]. In this paper, ie [40], three different natural convection problems, including nanofluid heat transfer problems, had been used to validate the numerical method. At this section, MHD natural convection in a cold enclosure with a hot inner cylinder filled with nanofluid has been investigated as the validation problem. In this problem the effect of magnetic field on natural convection has been considered. The details of the problem have been described in [47]. Figure 3 shows the average Nusselt number along the cold wall surface with respect to the aspect ratio $(\lambda)$. Also, Fig. 4 shows the $\mathrm{Nu}_{\text {ave }}$ on the surface of the hot cylinder with respect to the aspect ratio $(\lambda)$. Good agreement between the results presented by Sheikholeslami et al. [47] and the described numerical method results can be observed.

Furthermore, the grid independence evaluation of this study has been performed, which shows that the $210 \times 210$ uniform grid is an appropriate grid size. The grid independence study has been preformed for $\lambda=4.5$ and $\mathrm{Ra}=10^{6}$. The results of the grid evaluation are presented in Table 2 .

\section{Results and discussion}

In this section, the numerical solution results, consisting temperature contours and streamlines for five different Rayleigh numbers (i.e., $R a=10^{3}, 10^{4}, 10^{5}, 10^{6}$, and $10^{7}$ ), five different geometrical shape parameters, and the Hartmann numbers of $\mathrm{Ha}=0,50$ and 100 are presented in Sect. 6.1. Note that the isotherm contours and streamlines are presented for a fixed nano-particle volume fraction of $\varphi=2.5 \%$ (i.e., at the central point). Also, the effects of above-mentioned parameters on the average Nusselt number are investigated. Section 6.2 provides an equation 
T: 0.10 .20 .30 .40 .50 .60 .70 .80 .91

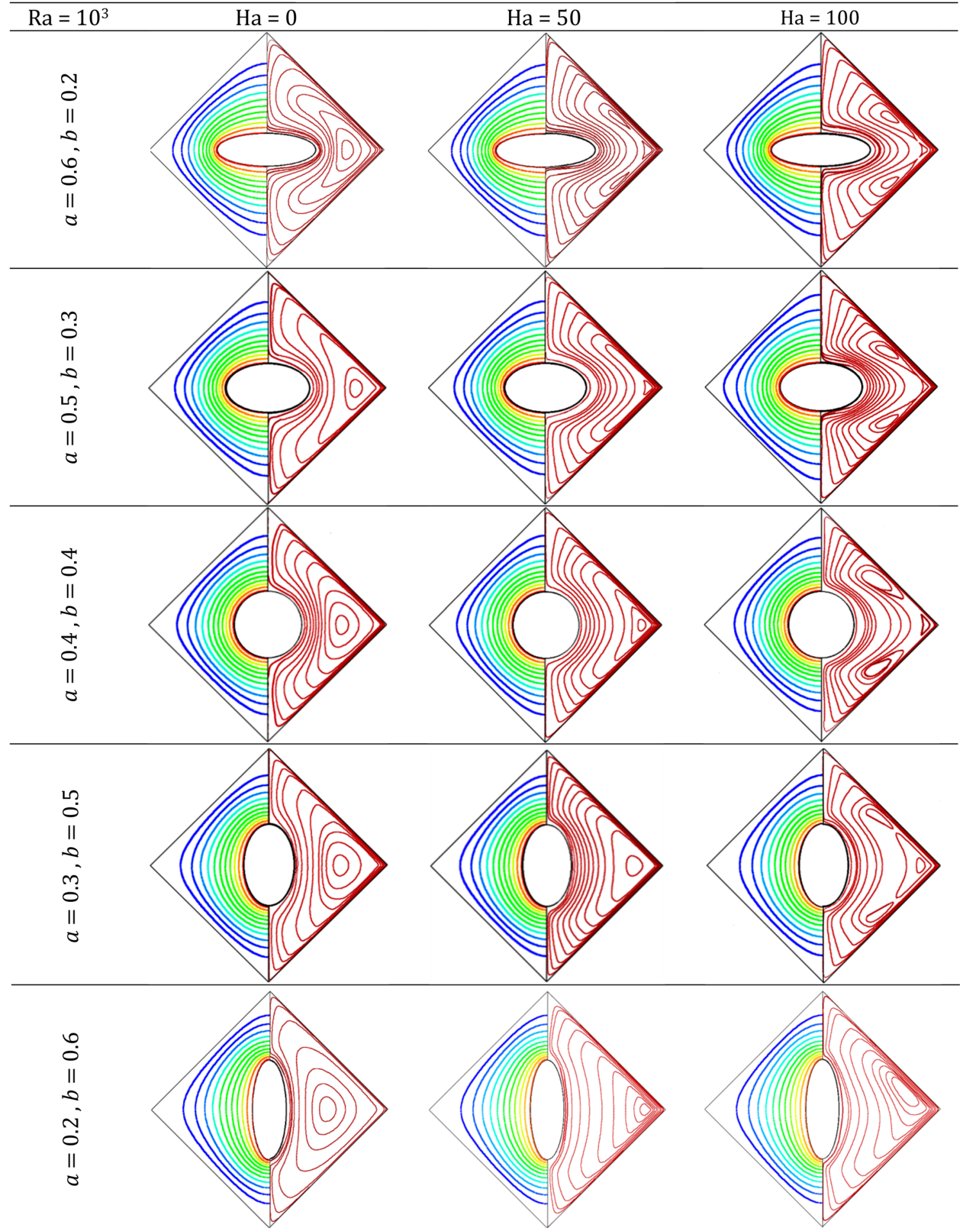

Fig. 5 Streamline and isotherms with respect to $\mathrm{Ra}=10^{3}$ 


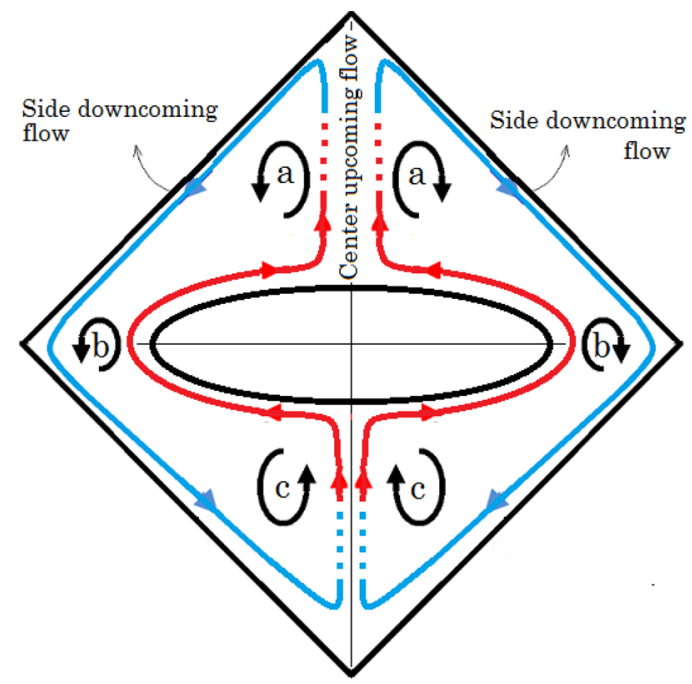

(a)

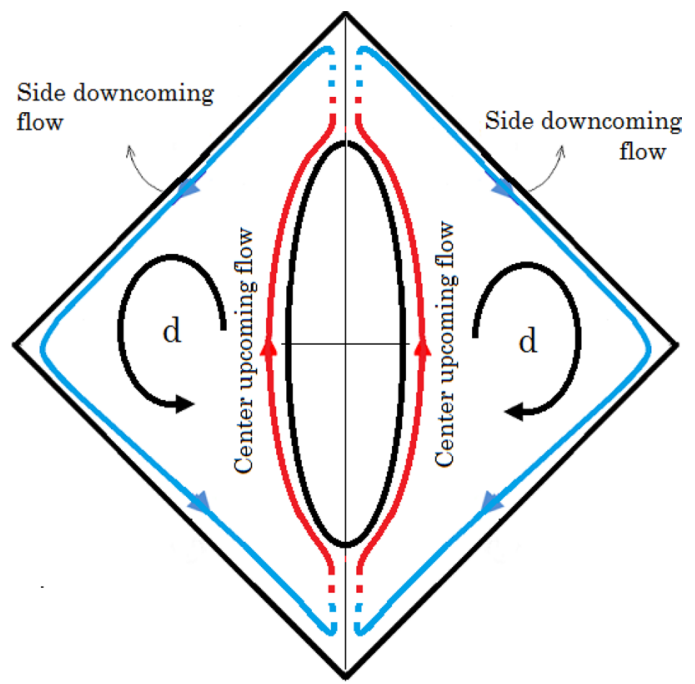

(b)

Fig. 6 Two patterns of CUF and SDF flows and the corresponding vortex locations

for the average Nusselt number based on the statistical regression of the numerical results.

\subsection{Numerical results}

Here, streamlines and isotherm contours are presented in five different Rayleigh numbers. Results show a symmetrical pattern of streamlines and isotherms in Rayleigh numbers of $\mathrm{Ra}=10^{3}, 10^{4}, 10^{5}$ and $10^{6}$. Therefore, in the presented following figures, half-field geometry is allocated to the streamlines and the other half to the isotherms. Figure 5 presents the streamlines and isotherms in the Rayleigh number of $\mathrm{Ra}=10^{3}$ in different geometrical shapes and for the Hartmann numbers of $\mathrm{Ha}=0,50$ and 100. In all images, $\varphi=2.5 \%$ is assumed to be constant. In this Rayleigh number, the conduction heat transfer is quite dominant and the effects of convectional heat transfer could be neglected. The isotherm lines, which play an important role in the heat transfer rate and the average Nusselt number, are independent of the Hartmann number in almost all cases. However, the magnetic field has a significant effect on the shape of the streamlines. In other words, in Rayleigh number of $R a=10^{3}$, magnetic field showed to be effective on the streamlines, and not on the isotherms. Examining the streamlines in Fig. 5, there are generally two types of streamlines, which are shown in Fig. $6 \mathrm{a}$ and b. Each of these two structures might be dependent up on the geometry, magnetic field strength, Rayleigh number, and volume fraction of the nanofluid. These two structures of the streamlines are as follows: (1) center up-coming flow (CUF) that flows upward from the hot cylindrical surface; and (2) side down-coming flow (SDF) that flows downward from the cold wall. These two flows play an important role in the formation of vortexes and the pattern of streamlines. A variation of the intensity of CUF and SDF flows might result in weakening, strengthening or removal of either vortexes of $a, b, c$, or d (Fig. 6). In the streamline structure shown in Fig. 6a, a maximum of three vortexes $a, b$ and $c$ could be formed. However, in the streamline structure of Fig. 6b, a maximum of one vortex, denoted by $\mathrm{d}$, might be formed. The location of these vortexes also significantly depends on the intensity of CUF and SDF flows as well as how the magnetic field is applied. Referring Fig. 5 , in $\mathrm{Ha}=100$, for the geometries of $(\mathrm{a}=0.3 / \mathrm{b}=0.5)$, $(a=0.4 / b=0.4),(a=0.5 / b=0.3),(a=0.6 / b=0.2)$, and also in $\mathrm{Ha}=50$, for the geometry of $(\mathrm{a}=0.6 / \mathrm{b}=0.2)$, a structure similar to Fig. $6 a$ is obvious. It is worth mentioning that in the Rayleigh number of $\mathrm{Ra}=10^{3}$, the isotherm lines are independent of the streamline curves shape due to the fully dominance of the conduction. Therefore, in all the presented modes, the isotherms have an almost constant shape. On the other hand, the structure shown in Fig. 6b is mostly formed in the lower Hartmann numbers and the geometries in which the oval is placed vertically. In these structures, only a large vortex, $d$, could be formed. The CUF flow is placed in the middle, and the SDF flows downward. Referring Fig. 5, in the Hartmann number of $\mathrm{Ha}=0$, in all geometries a structure similar to Fig. $6 \mathrm{~b}$ is observed. As well, in $\mathrm{Ha}=50$ for the geometries of $(a=0.2 / b=0.6)$, $(a=0.3 / b=0.5),(a=0.4 / b=0.4),(a=0.5 / b=0.3)$ a similar structure happens. Furthermore, the size of the formed vortexes depends upon the problem geometry and Hartmann number. Where the oval is horizontal, the vortex $b$ size is smaller and the vortexes a and $c$ are larger. On the 


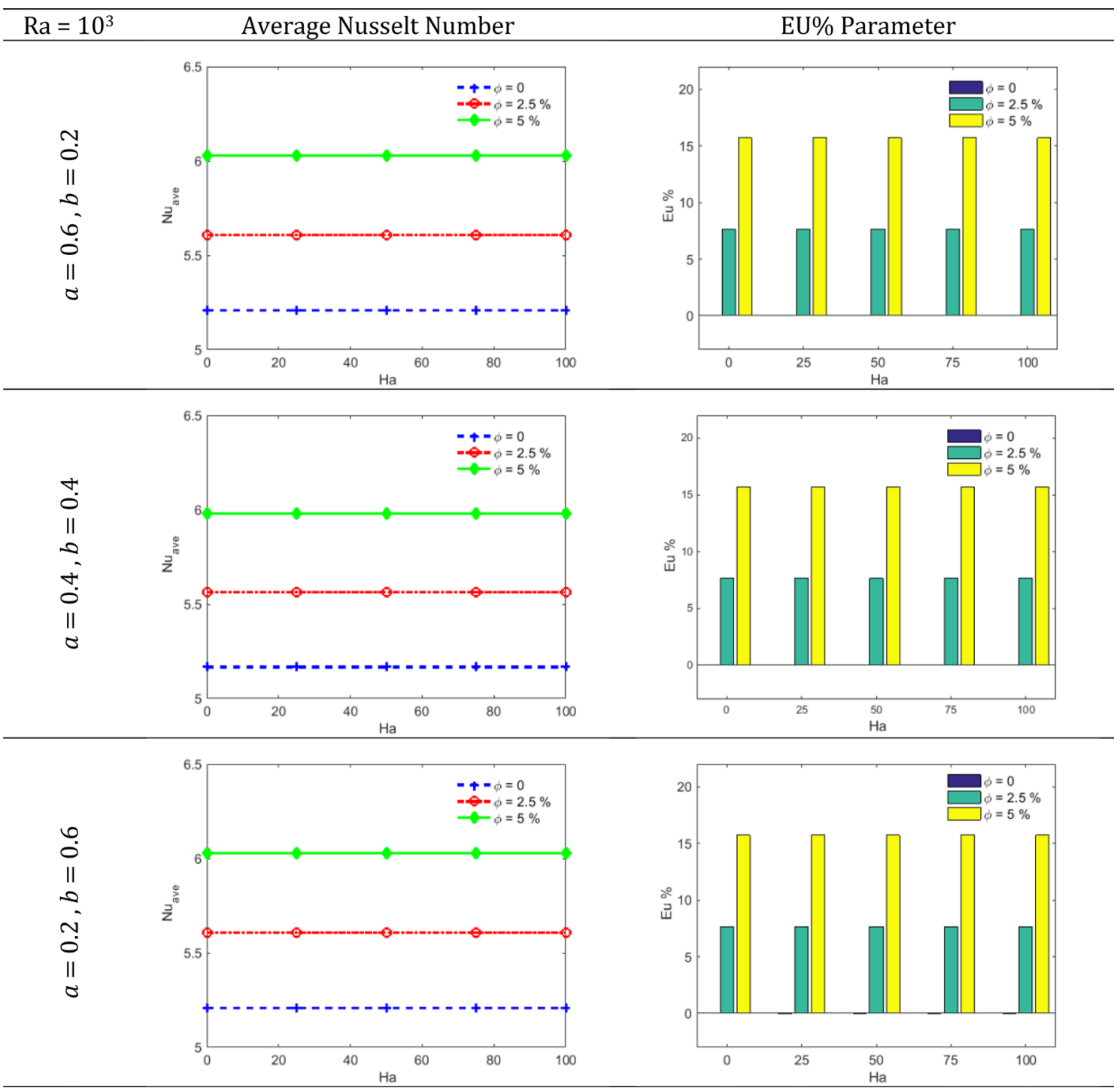

Fig. 7 Average Nusselt number and $\mathrm{EU} \%$ with respect to $\mathrm{Ra}=10^{3}$ for three different geometries

contrary, the vortex $b$ size would tend to be larger where the oval is vertical.

Figure 7 shows the average Nusselt number in three different geometries for threevolume fractions of nanofluid relative to the Hartmann number. The Eu\% parameter is also reported in each case. This parameter is defined in each Rayleigh number as follows:

$\mathrm{Eu} \%=\frac{\mathrm{Nu}_{\mathrm{ave}}(\varphi, \mathrm{Ha})-\mathrm{Nu}_{\mathrm{ave}}(\varphi, \mathrm{Ha})}{N u_{\mathrm{ave}}(\varphi, \mathrm{Ha})} \times 100$

Eu\% parameter determines how the presence of nanofluid and magnetic field, with respect to a constant geometrical parameter and Rayleigh number, effect on the average Nusselt number and consequently on the heat transfer value. As shown in Fig. 7, in $\mathrm{Ra}=10^{3}$, the Nusselt number is almost independent of the problem geometry and Hartmann number; however, as the nanofluid volume fraction increases its value enhances. This is due the fact that in $\mathrm{Ra}=10^{3}$, the conduction heat transfer is quite dominant. Thus, the convectional heat transfer does not play an effective role and as a result, the Hartmann number, which mainly influences on the streamlines, has a neglected effect. On the other hand, increasing the nanofluid volume fraction increases the conduction coefficient, which increases the total thermal conductivity and consequently increases the average Nusselt number. Calculations show that in $\mathrm{Ra}=10^{3}$ and in the case of $\varphi=5 \%$, the Nusselt number increased by $15.3 \%$ compared to the non-nanofluid state. 
$\mathrm{T:} \quad 0.10 .20 .30 .40 .50 .60 .70 .80 .91$

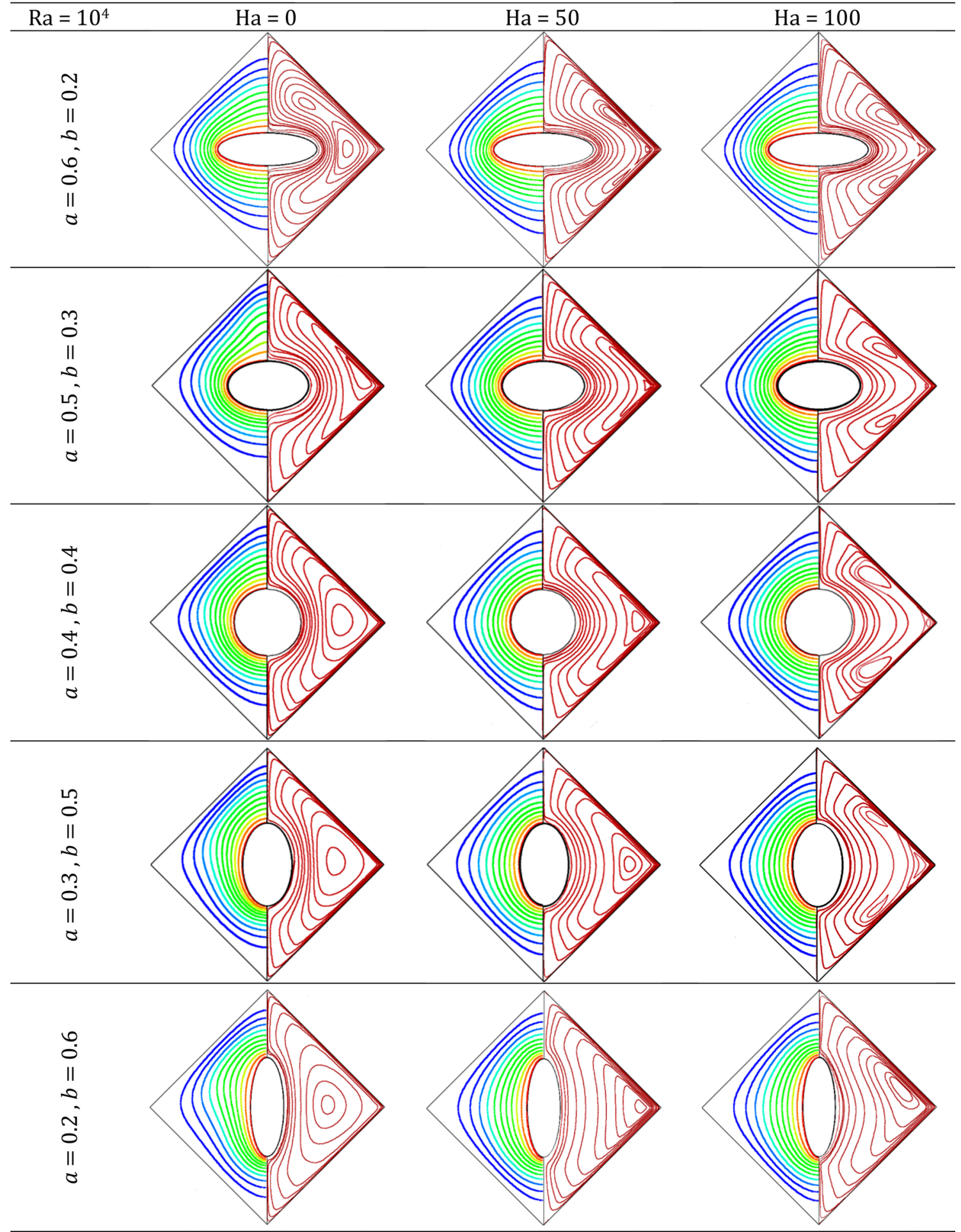

Fig. 8 Streamline and isotherms with respect to $\mathrm{Ra}=10^{4}$

As the Rayleigh number increases to $\mathrm{Ra}=10^{4}$, the role of convectional heat transfer increases, but the conduction is still dominant. Figure 8 illustrates the streamlines and isotherms in this Rayleigh number. Here, in $\mathrm{Ha}=100$ for the geometries of $(a=0.3 / b=0.5),(a=0.4 / b=0.4)$, $(a=0.5 / b=0.3),(a=0.6 / b=0.2)$ the flow structure is similar 


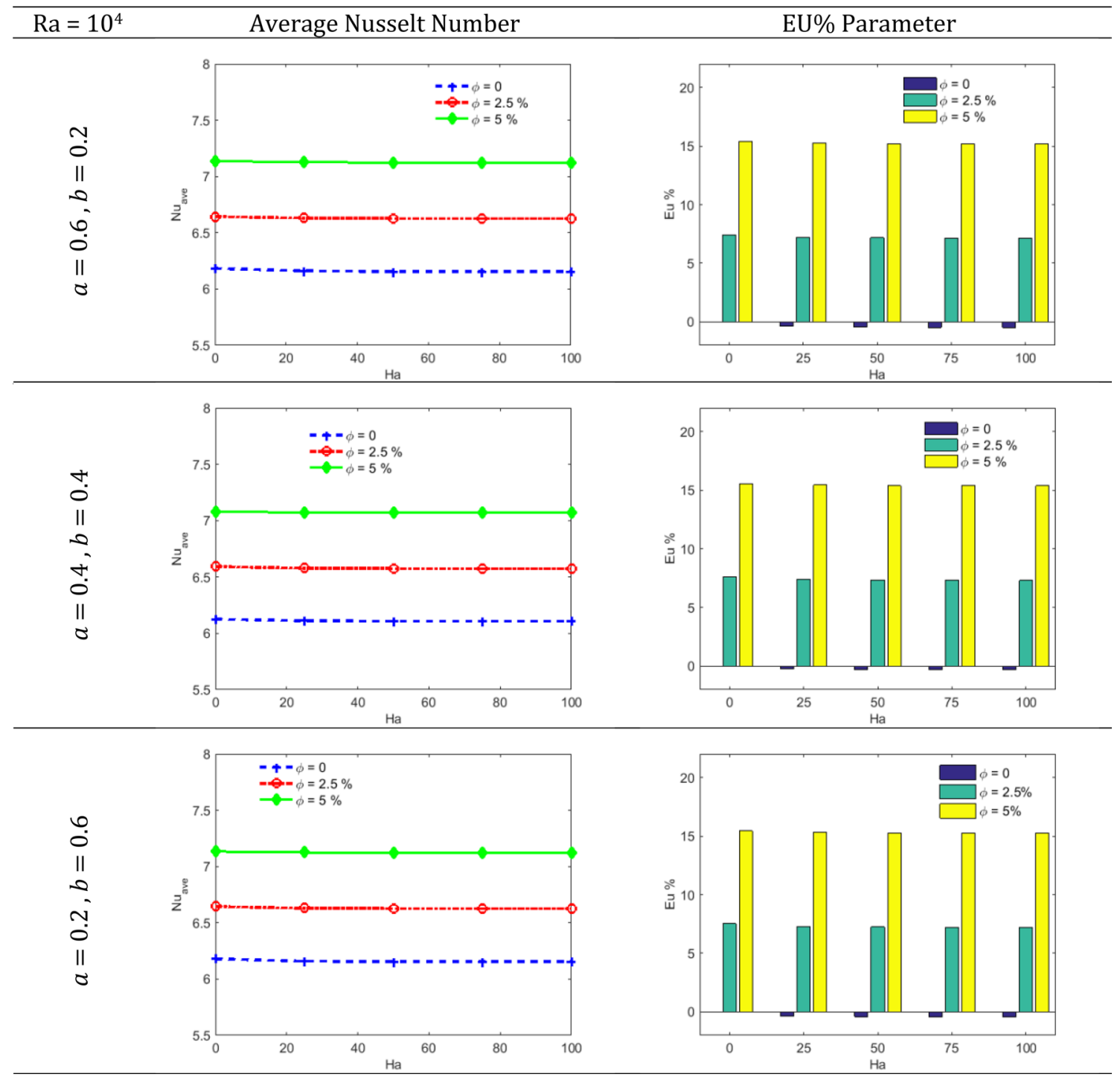

Fig. 9 Average Nusselt number and EU\% with respect to $\mathrm{Ra}=10^{4}$ for three different geometries

to Fig. 6a. However, it would weaken or strengthen the vortexes $\mathrm{a}, \mathrm{b}$ and $\mathrm{c}$ in proportion to the problem geometry. As well, for the geometry of $(a=0.2 / b=0.6)$ the flow structure is similar to Fig. $6 \mathrm{~b}$. In $\mathrm{Ha}=50$, the flow structure of Fig. $6 \mathrm{a}$ is created in the geometry of $(a=0.6 / b=0.2)$. As the CUF flow intensifies here, a larger vortex $a$ is formed at the upper surface of the oval. Note that in $\mathrm{Ha}=50$, the rest of the geometrical states show the flow structure similar to Fig. $6 \mathrm{~b}$. Finally, in $\mathrm{Ha}=0$, when the magnetic field has no effect on the flow, a similar structure to Fig. $6 \mathrm{a}$ is created at the geometry of $(a=0.6 / b=0.2)$. However, the vortex $c$ is so weakened or practically eliminated due to the strengthening of CUF flow. Other geometrical parameters at the same Hartmann number would be similar to Fig. 6b.As mentioned, in $\mathrm{Ra}=10^{4}$, the conduction heat transfer is practically dominant. The isotherms are almost symmetrical around the hot cylinder and there is not much difference between them. It worth mentioning that at Hartmann numbers of $\mathrm{Ha}=50$ and $\mathrm{Ha}=100$ the isotherms are ideally symmetrical. While, at $\mathrm{Ha}=0$ the isotherms are slightly upward, indicating a relative increase in the convectional heat transfer role.

Figure 9 presents the average Nusselt number and EU\% in three different geometries for three nanofluid volume fractions relative to the Hartmann number. As shown, in $\mathrm{Ra}=10^{4}$, the Nusselt number is very little affected by the Hartmann number. However, as the nanofluid volume fraction increases $\mathrm{Nu}_{\text {ave }}$ significantly increases. Also, the Nusselt number is almost independent of the geometrical shape. This is due to the fact that in $\mathrm{Ra}=10^{4}$, the conduction heat transfer is relatively dominant. Hence, convection does not play an important role in this regard, so the Hartmann number has a neglected effect on Rayleigh number. On the other 
T: 0.10 .20 .30 .40 .50 .60 .70 .80 .91

\begin{tabular}{|c|c|c|c|}
\hline $\mathrm{Ra}=10^{5}$ & $\mathrm{Ha}=0$ & $\mathrm{Ha}=50$ & $\mathrm{Ha}=100$ \\
\hline $\begin{array}{l}N \\
0 \\
I I \\
0 \\
0 \\
0 \\
0 \\
\| \\
0\end{array}$ & & & \\
\hline $\begin{array}{l}m \\
0 \\
\| 1 \\
0 \\
0 \\
0 \\
0 \\
\| \\
0\end{array}$ & & & \\
\hline $\begin{array}{l}\ddot{H} \\
0 \\
\| 1 \\
0 \\
\vdots \\
0 \\
0 \\
\| \\
0\end{array}$ & & & \\
\hline $\begin{array}{l}\text { ?n } \\
0 \\
11 \\
0 \\
n \\
0 \\
0 \\
11 \\
0\end{array}$ & & & \\
\hline $\begin{array}{l}0 \\
0 \\
\| 1 \\
0 \\
01 \\
\tilde{0} \\
\| \\
\| \\
0\end{array}$ & & & \\
\hline
\end{tabular}

Fig. 10 Streamline and isotherms with respect to $\mathrm{Ra}=10^{5}$

hand, increasing the nanofluid volume fraction increases the conduction coefficient, which increases the total thermal conductivity and as a result, increases the average
Nusselt number. Calculations show that in $\mathrm{Ra}=10^{4}$, in the case of $\varphi=5 \%$, the Nusselt number increased by $15.45 \%$ compared to the non-nanofluid state. Also, as Hartmann 
number is equal to 100 , in the case of $\varphi=0 \%$, the maximum amount of Nusselt number reduction by $1.2 \%$ occurs, compared to the state without applying a magnetic field.

In Rayleigh number of $\mathrm{Ra}=10^{5}$, the role of conduction and convection heat transfer at $\mathrm{Ha}=100$ are comparable. Figure 10 illustrates the streamlines and isotherms with respect to $\mathrm{Ra}=10^{5}$. In this Hartmann number, in all cases except the $(a=0.2 / b=0.6)$ a structure similar to Fig. $6 a$ is obvious. However, vortex $a, b$, and $c$ intensities are in proportion to the problem geometry. For example, in $\mathrm{Ha}=100$, in the case of $(\mathrm{a}=0.6 / \mathrm{b}=0.2)$, all three vortexes $a, b$ and $c$ are available, but vortexes $a$ and $c$ is larger than vortex $b$. In the case of $(a=0.5 / b=0.3)$ the vortex $b$ is weakened so that is practically not visible in the flow field. In the case of $(a=0.4 / b=0.4)$ vortex $b$ is formed again in the flow field and on the contrary, the vortex $c$ is somewhat weakened. In the case of $(a=0.3 / b=0.5)$ vortex $b$ is strengthened and vortexes $a$ and $c$ are weakened. Finally, in the case of $(a=0.2 / b=0.6)$ practically the same structure to Fig. $6 \mathrm{~b}$ could be seen. In $\mathrm{Ha}=50$, in the geometry of $(a=0.6 / b=0.2)$ only a large vortex $a$ is created on the upper surface of the hot cylinder, according to the structure Fig. 6 a, by intensifying the CUF flow. Similarly, this could be seen in the state of $(a=0.5 / b=0.3)$. In the state of $(a=0.4 / b=0.4)$ vortex $b$ is amplified, thus both vortexes $a$ and $b$ are available. However, in two modes of $(a=0.3 / b=0.5)$ and $(a=0.2 / b=0.6)$ a similar structure of Fig. $6 \mathrm{~b}$ is created. In this structure, vortex $\mathrm{d}$ tends to move upward due to the increased CUF flow. Finally, In $\mathrm{Ha}=0$, in two modes of $(\mathrm{a}=0.6 / \mathrm{b}=0.2)$ and $(a=0.5 / b=0.3)$ a structure similar to Fig. $6 a$ is created, but only vortexes $a$ and $b$ are visible and no vortex $c$ is created

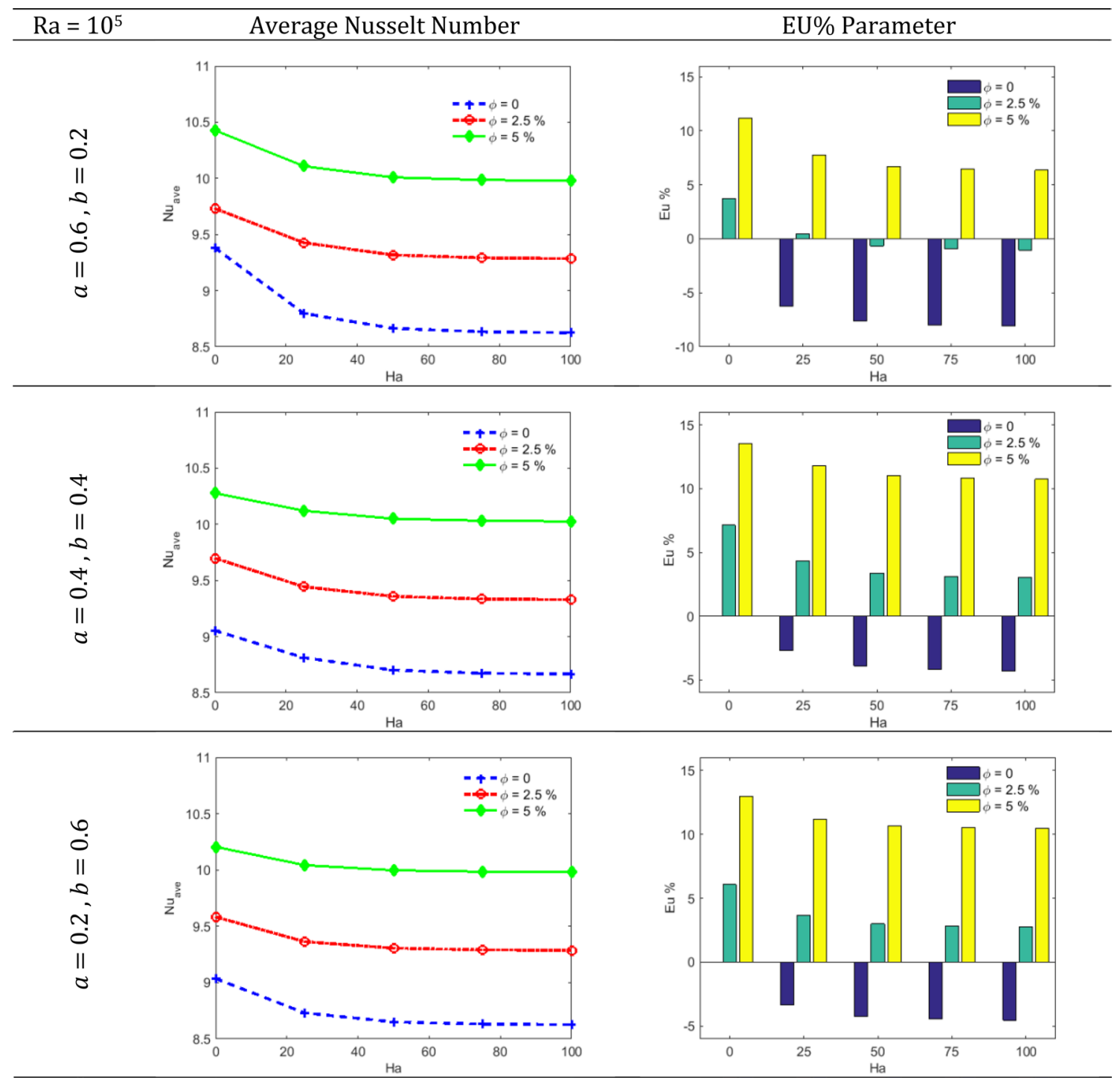

Fig. 11 Average Nusselt number and EU\% with respect to Ra $=10^{5}$ for three different geometries 
$\mathrm{T:} \quad 0.10 .20 .30 .40 .50 .60 .70 .80 .91$

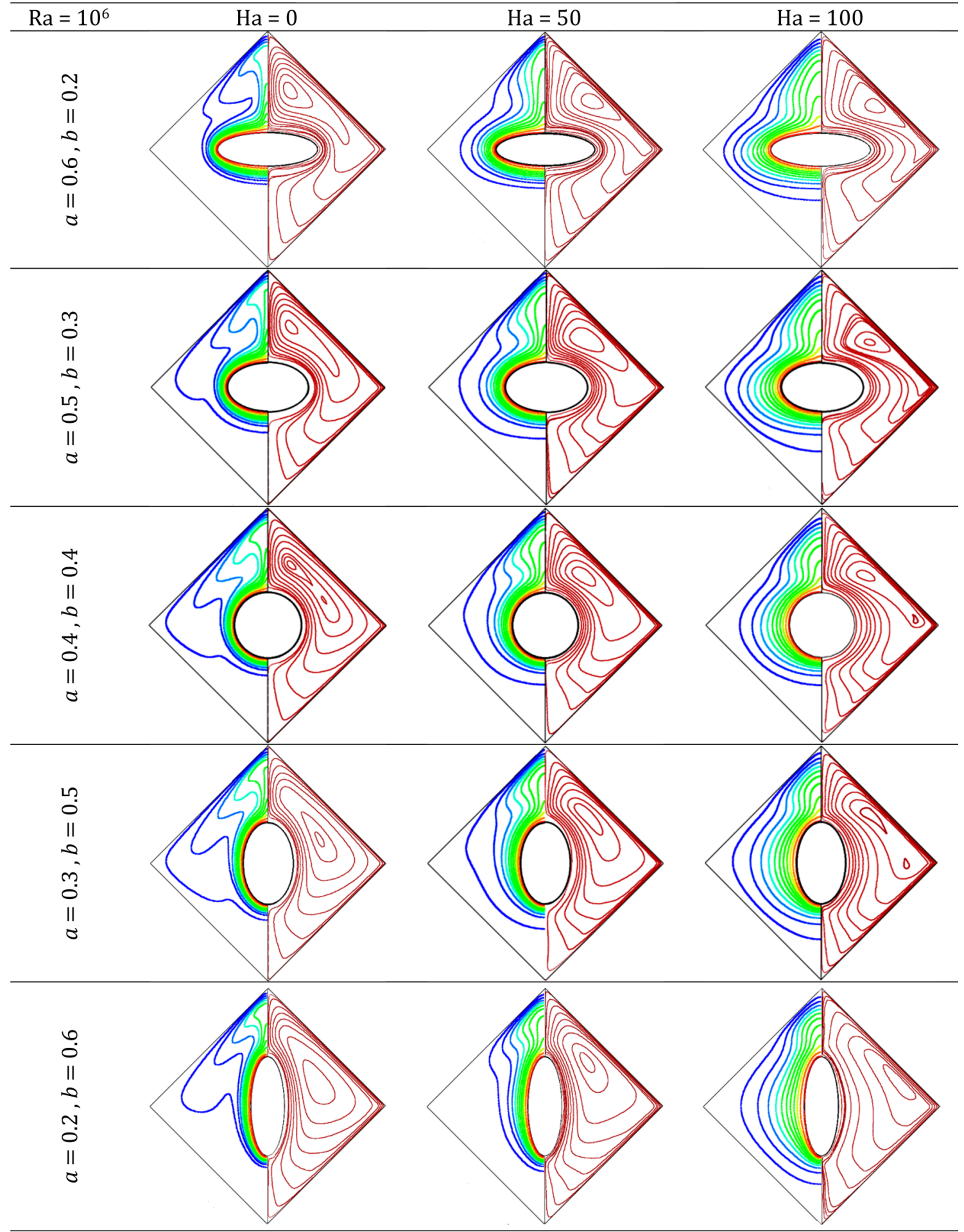

Fig. 12 Streamline and isotherms with respect to $\mathrm{Ra}=10^{6}$

due to the strengthening of the CUF flow. In the state of $(a=0.6 / b=0.2)$ vortex a would be larger than vortex $b$. While, in the mode of $(a=0.5 / b=0.3)$ vortex $a$ is weakened and vortex $b$ is strengthened. In all other cases, a structure similar to Fig. $6 \mathrm{~b}$ is created. The isotherms would also have a different shape at $\mathrm{Ra}=10^{5}$ as the convectional heat 
transfer is amplified. At Hartmann number of $\mathrm{Ha}=100$, the isotherms tend to displace slightly upward and are almost ideally symmetrical. This indicates that the effect of the magnetic field is a significant effect. In fact, applying a magnetic field weakens the CUF flow and reduces the role of convectional heat transfer. By reducing the intensity of the magnetic field to $\mathrm{Ha}=50$, the CUF flow is amplified and the isotherms lines tend to move towards the upper part of the enclosure. As the magnetic field is stopped, $\mathrm{Ha}=0$, the convectional heat transfer role becomes prominent and creates completely columnar lines toward the upper part of the enclosure.

Figure 11 shows the distribution of the Nusselt number and EU\% relative to the Hartmann number in different geometries and for different nanofluid volume fractions. As is obvious, in $\mathrm{Ra}=10^{5}$, with the increase of $\mathrm{Ha}$ in all cases, the average Nusselt number has a decreasing trend. Also, with increasing $\varphi$ the heat transfer rate has increased, compared to $\varphi=0$. The highest increase in $\mathrm{Nu}_{\text {ave }}$ is equal to $13.8 \%$ in the state of $(a=0.4 / \mathrm{b}=0.4)$, where $\mathrm{Ha}=0$ and $\varphi=5 \%$. On the other hand, the highest decrease in the Nusselt number is equal to $-8.15 \%$ in the mode of $(a=0.6 / b=0.2)$, where $\mathrm{Ha}=100$ and $\varphi=0$. In the same mode, where $\varphi=2.5 \%$ and for the Hartmann numbers of 50,75 and 100, the effect of magnetic field is more significant than the presence of nanofluid; this causes the Eu\% parameter to have a negative value. However, in all other cases, the effect of nanofluid volume fraction has been augmented, and therefore Eu\% has a positive value.

As the Rayleigh number changes to $R a=10^{6}$, the CUF flow intensity augments and thus, the convectional heat transfer is quite dominant. Figure 12 shows the streamlines

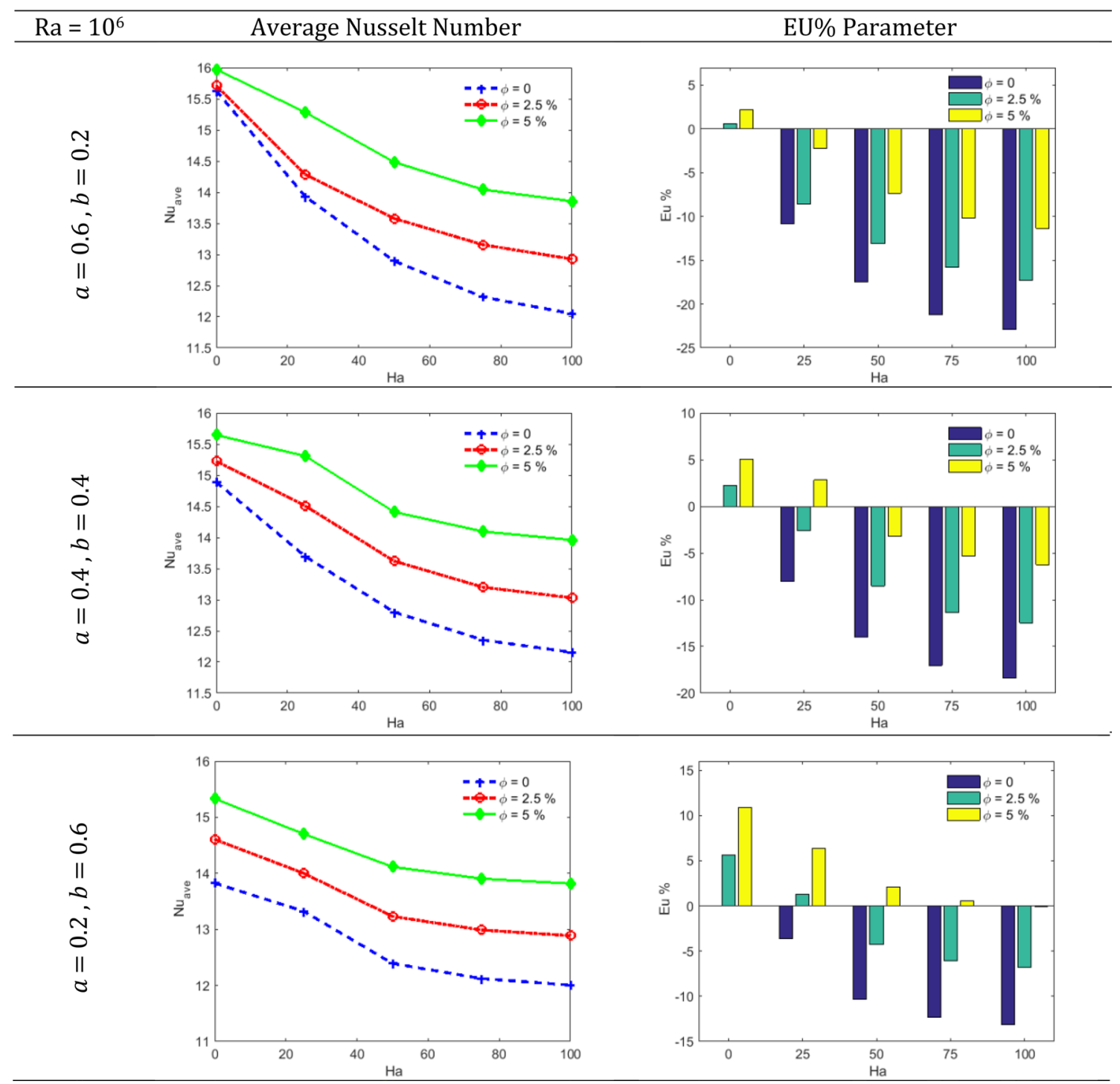

Fig. 13 Average Nusselt number and EU\% with respect to $\mathrm{Ra}=10^{6}$ for three different geometries 
and isotherms with respect to $\mathrm{Ra}=10^{6}$. In $\mathrm{Ha}=100$, although there is a strong magnetic field, the intensity of the CUF causes the vortex a to be observed in two states of $(a=0.6 / b=0.2)$ and $(a=0.5 / b=0.3)$. However, a weak vortex of $b$ is also available in the modes $(a=0.4 / b=0.4)$ and $(a=0.3 / b=0.5)$. On the other hand, a structure of Fig. $6 b$ is formed in the mode of $(a=0.2 / b=0.6)$. In Ha=50, the effect of magnetic field on the streamlines is reduced. Therefore, CUF flow has the main role in this regard. In all geometries, only a large vortex is created at the top of the hot cylinder, which tends to move upwards. In the case without applying a magnetic field $(\mathrm{Ha}=0)$, convection heat transfer is quite dominant. Here, again in all geometries, only one large vortex is created at the top of the hot cylinder. However, in the mode of $(a=0.4 / b=0.4)$ a very small secondary vortex is also visible. It is worth mentioning that in Hartmann number equal to zero, the vortexes are formed exactly on the upper surface of the hot cylinder. While, in $\mathrm{Ha}=50$ and 100 the vortexes are slightly inclined to the right on the upper surface of the cylinder. Furthermore, In $\mathrm{Ha}=0$, a structure similar to Fig. $6 a$ is formed in the modes of $(a=0.6 / b=0.2),(a=0.5 /$ $b=0.3)$ and $(a=0.4 / b=0.4)$, which shows the sole vortex a due to the high intensity of the CUF flows. In the states of $(a=0.3 / b=0.5)$ and $(a=0.2 / b=0.6)$ a pattern of Fig. $6 b$ is created, in which the vortex $d$ tends upwards. The isotherms in $\mathrm{Ha}=50$ and 100 tend to be completely upward, and could be seen as a column on the upper surface of hot cylinder. Also, in $\mathrm{Ha}=50$ the slope of isotherm lines are steeper. In $\mathrm{Ha}=0$, the isotherm lines are mushroomshaped and are placed around the hot cylinder. The flow intensity is also higher in this case due to the absence of a magnetic field.

Figure 13 illustrates the average $\mathrm{Nu}_{\mathrm{ave}}$ and $\mathrm{Eu} \%$ parameter in three different geometries with respect to $\mathrm{Ra}=10^{6}$. In all cases, Nusselt number shows a decreasing trend as $\mathrm{Ha}$
Fig. 14 Streamline and isotherms with respect to $\mathrm{Ra}=10^{7}$ for $\mathrm{Ha}=50$ and 100
T: $\quad 0.10 .20 .30 .40 .50 .60 .70 .80 .91$

$\mathrm{Ra}=10^{7}$


Fig. 15 Streamline and isotherms with respect to $\mathrm{Ra}=10^{7}$ for $\mathrm{Ha}=0$

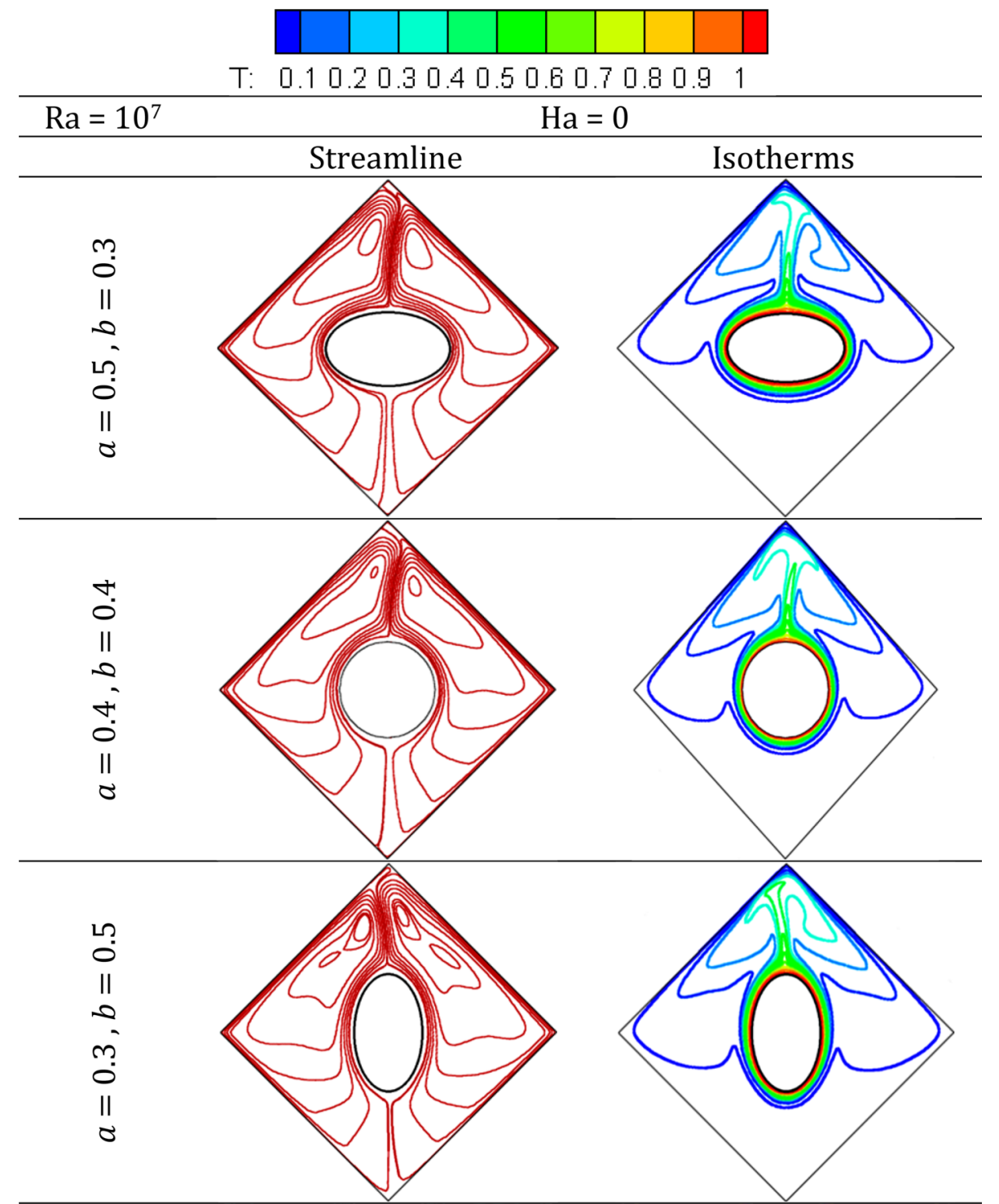

changes. In fact, applying a magnetic field reduces the intensity of the flow generated by the convectional heat transfer, and this leads to a reduction in the overall rate of heat transfer. Furthermore, increasing the nanofluid volume fraction increases the $\mathrm{Nu}_{\text {ave }}$ in all cases, and enhances the total heat transfer rate. The highest Nusselt number occurs in the most vertical geometry (where $a=0.6$ and $b=0.2$ ). As the problem geometry changes from the horizontal extreme to the vertical one, the average Nusselt number increases; because when the convectional heat transfer is predominant, the cross area of the flow is higher in the vertical geometry, and hence the overall rate of heat transfer increases.

Figure 13 also shows that nanofluid volume fraction and the Hartmann number have reverse influences on Nusselt number. For example, in the case of $(a=0.6 / b=0.2)$ and $\mathrm{Ha}=25,50,75$ and 100 in all cases, the effect of magnetic field is more significant than nanofluid volume fraction, which leads to a negative Eu\% parameter. Similarly, in the case of $(\mathrm{a}=0.4 / \mathrm{b}=0.4)$ in $\mathrm{Ha}=50,75$ and 100 , Eu\% parameter is still negative in all cases. However, for the former mode and, where $\mathrm{Ha}=25$ and $\varphi=5 \%$ the effect of nanofluid volume fraction increase and Eu\% parameter becomes positive. This fades in $\varphi=2.5 \%$, and $\mathrm{Eu} \%$ experiences negative values again. As well, in the case of $(a=0.2 / b=0.6)$ and $\varphi=5 \% \mathrm{Eu} \%$ is a positive value, while for the $\varphi=2.5 \%$ and $\mathrm{Ha}=50,75$ and $100 \mathrm{Eu} \%$ is negative. According to the numerical results, the highest increase in Nusselt number is equal to $11.2 \%$ in the mode of $(a=0.2 / b=0.6)$, when $\varphi=5 \%$ and $\mathrm{Ha}=0$. The highest decrease is also equal to $23.4 \%$ and is observed in the mode of $(a=0.6 / b=0.2)$, when $\varphi=0$ and $\mathrm{Ha}=100$.

Figure 14 shows the streamlines and isotherms with respect to $\mathrm{Ra}=10^{7}$, for two Hartmann numbers of $\mathrm{Ha}=50$ and 100. The similar curves and contours for $\mathrm{Ha}=0$ in the 


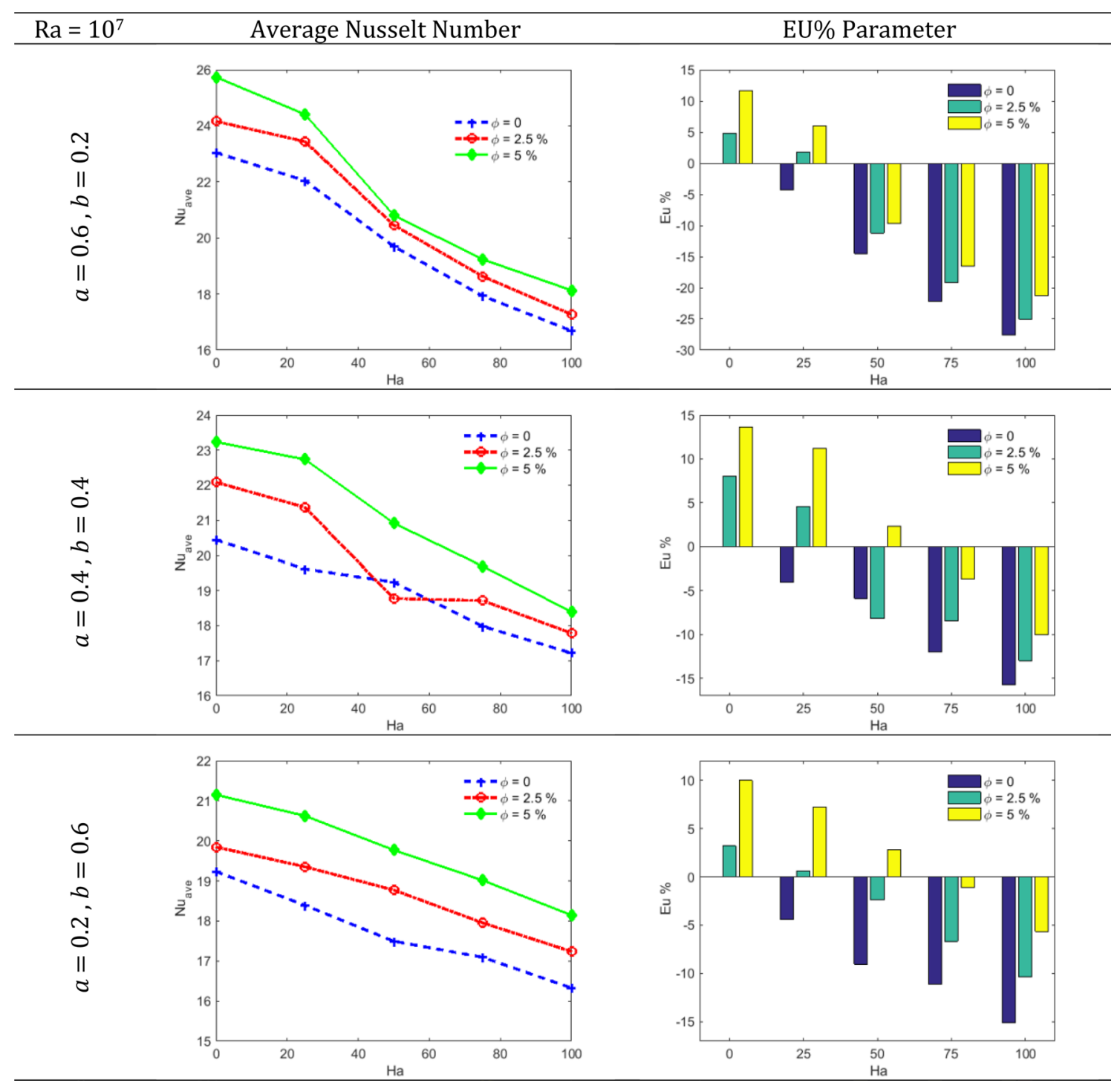

Fig. 16 Average Nusselt number and EU\% with respect to $\mathrm{Ra}=10^{7}$ for three different geometries

Table 3 Process parameters and the defined levels

\begin{tabular}{llll}
\hline Parameter & 1st level & Center point & 3rd level \\
\hline $\log (\mathrm{Ra})$ & 4 & 5 & 6 \\
$\varphi$ & 0 & 0.25 & 0.5 \\
$\mathrm{Ha}$ & 0 & 50 & 100 \\
$\mathrm{a}$ & 0.2 & 0.4 & 0.6 \\
$\mathrm{~B}$ & 0.2 & 0.4 & 0.6 \\
\hline
\end{tabular}

same Rayleigh number is also illustrated in Fig. 15. An important observation here is that in $\mathrm{Ra}=10^{7}$, by amplifying the intensity of the CUF at $\mathrm{Ha}=0$, the flow becomes asymmetric and unsteady. However, in $\mathrm{Ha}=50$ and 100 , by applying a magnetic field the symmetry of the flow would be recreated. In fact, in this Rayleigh number the convectional effect is predominant, so the rising columnar flow with high intensity overshadows the stability of the flow field and as a result, the symmetry of the flow is disturbed. On the other hand, when a magnetic field is applied to a flow field, the magnetic field induced forces stabilize again the flow field by weakening the upward flows.

In all cases shown in Fig. 14, the streamlines create only large vortexes on the upper surface of the hot cylinder. The isotherm lines also form a mushroom-like shapes on it. Figure 15 shows that an unsteadiness and asymmetry created as the magnetic field is absent. Here, the created vortexes at the upper surface of the hot cylinder shrink and expand, leads to an asymmetrical flow pattern.

Figure 16 presents the Nu number and the Eu\% parameter as a function of $\mathrm{Ha}$ for $\mathrm{Ra}=10^{7}$. In general, with 
Fig. 17 The effects of input parameters on the average Nusselt number

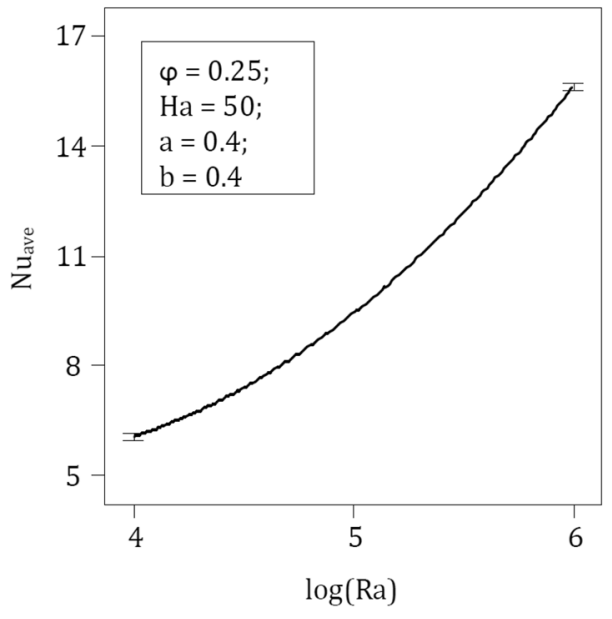

(a) effect of $\log (\mathrm{Ra})$ on $\mathrm{Nu}_{\text {ave }}$

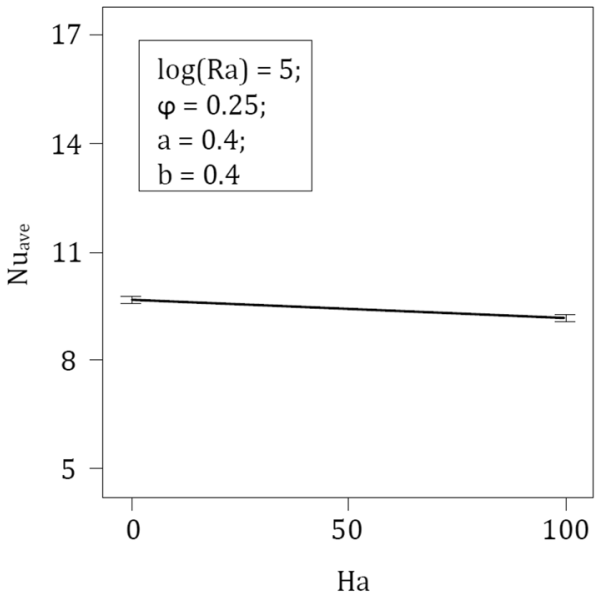

(c) effect of $\mathrm{Ha}$ on $\mathrm{Nu}_{\mathrm{ave}}$

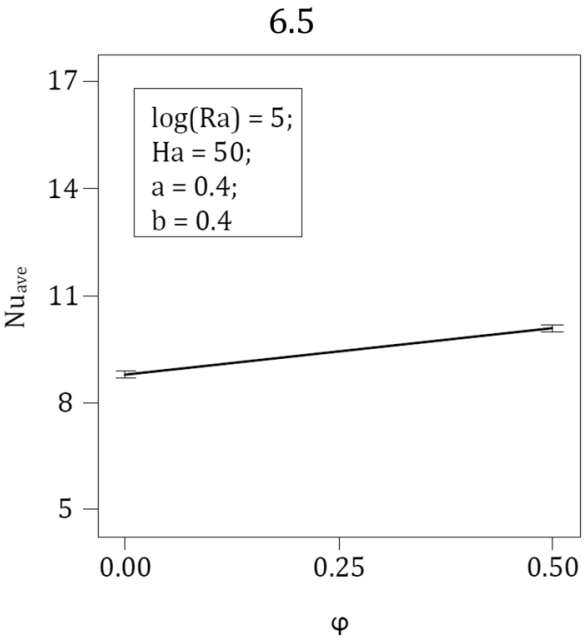

(b) effect of $\varphi$ on $\mathrm{Nu}_{\text {ave }}$

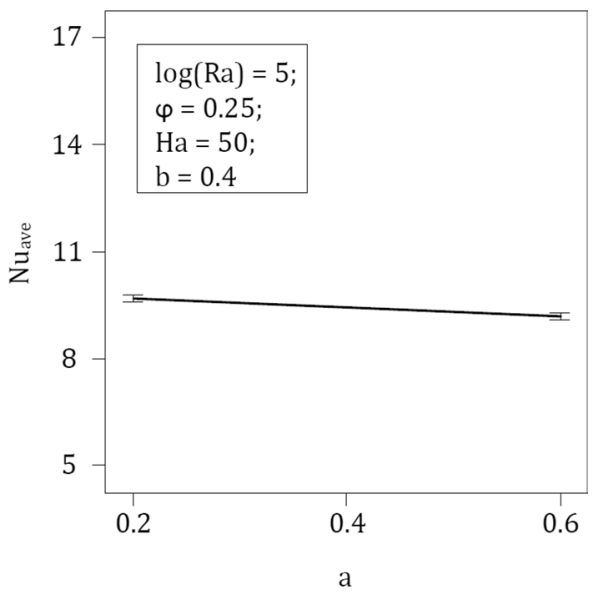

(d) effect of a on $\mathrm{Nu}_{\text {ave }}$

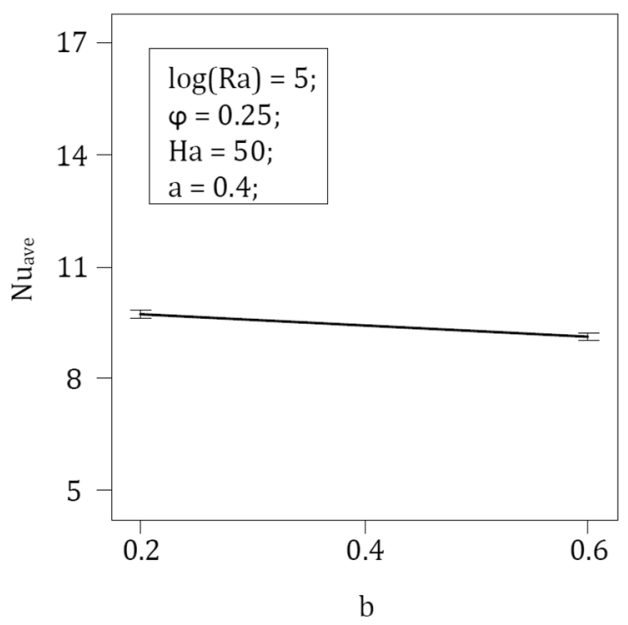

(e) effect of b on $\mathrm{Nu}_{\mathrm{ave}}$

increasing the Hartmann number, the Nusselt number and the total heat transfer rate decrease. The highest increase in $\mathrm{Nu}_{\mathrm{ave}}$ occurred in the mode of $(\mathrm{a}=0.4 / \mathrm{b}=0.4)$, and $\mathrm{Ha}=0$ and $\varphi=5 \%$ that is equal to $13.8 \%$. The highest decrease in the $\mathrm{Nu}_{\text {ave }}$ was also observed in the case of $(\mathrm{a}=0.6 / \mathrm{b}=0.2)$, $\mathrm{Ha}=100$ and $\varphi=0$ that is equal to -27.3. It is worth mentioning that in the case of $(a=0.4 / b=0.4)$ and $\mathrm{Ha}=50$, an increase in nanofluid volume fraction by $\varphi=2.5 \%$ leads to a 


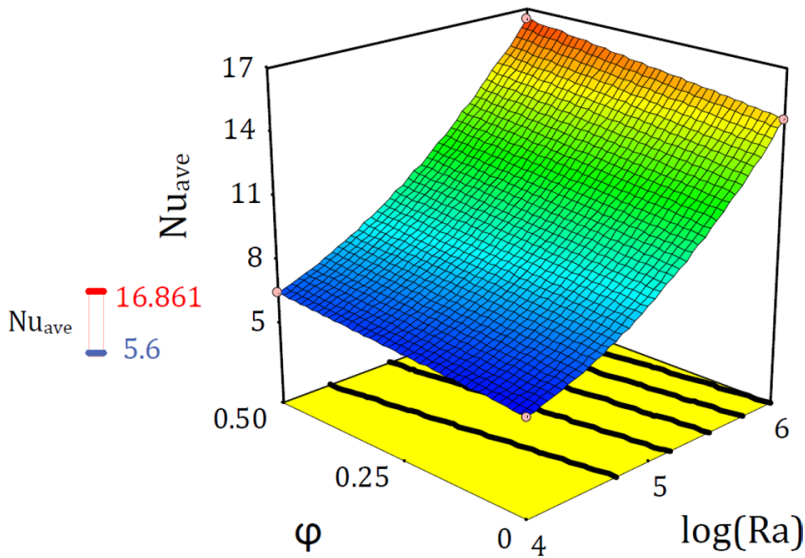

(a) effect of $\log (\mathrm{Ra})$ and $\varphi$ on $\mathrm{Nu}_{\mathrm{ave}}$

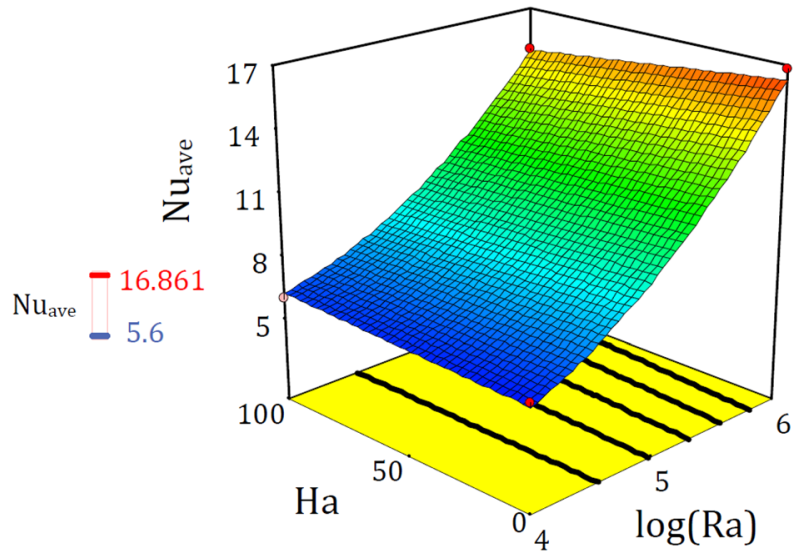

(b) effect of $\log (\mathrm{Ra})$ and $\mathrm{Ha}$ on $\mathrm{Nu}_{\mathrm{ave}}$

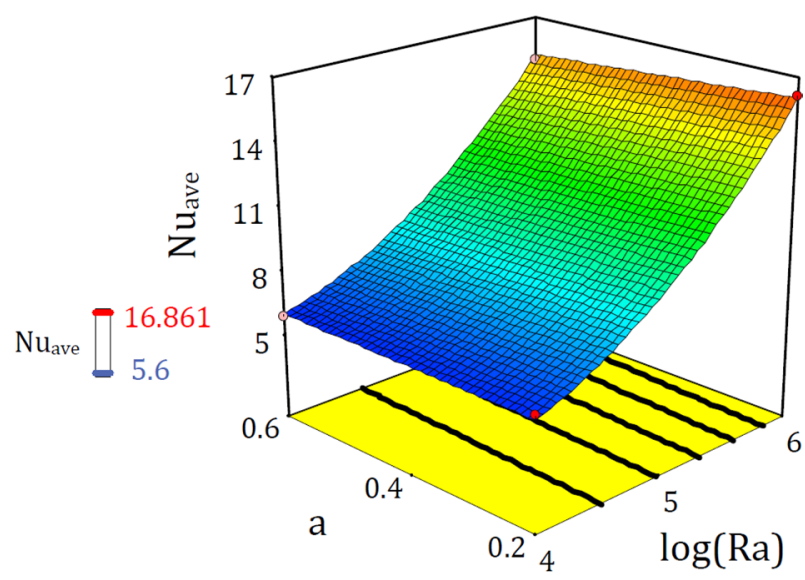

(c) effect of $\log (\mathrm{Ra})$ and a on $\mathrm{Nu}_{\text {ave }}$

Fig. 18 The interactive effects of input parameters on the average Nusselt number

decreased $\mathrm{Nu}_{\mathrm{ave}}$. But in other cases, an increase in nanofluid volume fraction leads to an increase in Nusselt number.

\subsection{Statistical analysis of the numerical results}

In this section, a Box-Behnken design of experiment is carried out to study the quantitative effect of several parameters on the average Nusselt number. The corresponding input parameters, which each is defined in three different levels, are listed in Table 3. Referring three levels for five parameters, and with consideration of one center point, the total 40 numerical simulations are performed. Analysis of Variance (ANOVA) of the numerical results showed that a linear model, along with the added significant $2 \mathrm{FI}$ and quadratic terms, is an appropriate equation to predict the average Nusselt number in the defined input parameter range, as follows:

$$
\begin{aligned}
N u_{\text {ave }}= & 18.05-8.65 \times \log (R a)-2.11 \times \varphi \\
& +0.04 \times \mathrm{Ha}+4.29 \times a-1.43 \times b \\
& +0.96 \times \log (R a) \times \varphi--0.009 \\
& \times \log (R a) \times \mathrm{Ha}-1.09 \times \log (R a) \\
& \times a+1.41 \times \log (R a)^{2}
\end{aligned}
$$

The effects of each parameter on the average Nusselt number are shown in Fig. 17a-e. There is a positive correlation between log (Ra) and $\varphi$ on $\mathrm{Nu}_{\text {ave }}$. In this regard, the $\log (\mathrm{Ra})$ has a more significant effect on the response; as log (Ra) changes from 4 to 6 , the $\mathrm{Nu}_{\text {ave }}$ is increased from 6.07 to 15.6. As well, Three other parameters, including $\mathrm{Ha}$, $\mathrm{a}$ and $\mathrm{b}$ shows a negative correlation. The interactive effects of input parameters on the response are illustrated in Fig. 18. Finally, the minimum $\mathrm{Nu}_{\text {ave }}$ of 5.52 is specified, by optimization of the presented model, as $\log (R a)=4$, $\varphi=0, \mathrm{Ha}=52, \mathrm{a}=0.42$, and $\mathrm{b}=0.51$. Maximum $\mathrm{Nu}_{\text {ave }}$ of 17.06 is specified as $\log (R a)=6, \varphi=0.47, \mathrm{Ha}=8, a=0.34$, and $b=0.30$.

\section{Conclusion}

This study uses an iterative direct forcing immersed boundary method to study the flow and heat transfer in a cold rhombus enclosure with a hot internal oval cylinder. The volume between the hot cylinder and cold enclosure is filled with a nanofluid, and the whole area is affected by the magnetic field. In this manuscript, the effect of Rayleigh number, Hartmann number, oval geometrical dimensions (i.e., small and large diameter), and nanofluid volume fraction were investigated. Also, by analysis of a set of numerical results a statistical model is extracted for the average Nusselt number. The most important results of the study could be summarized as follows: 
1. The iterative direct forcing immersed boundary method has been reliably developed in order to investigate the heat transfer problem.

2. In low Rayleigh numbers of $R a=10^{3}$ and $R a=10^{4}$, the effect of the Hartmann number on $\mathrm{Nu}_{\text {ave }}$ is negligible. Also, as $\varphi=5 \%$, Nusselt number would increase by $15.3 \%$ and $15.45 \%$, respectively.

3. In $\mathrm{Ra}=10^{5}$, as the Hartmann number increases the average Nusselt number would decrease. The highest decrease in $\mathrm{Nu}_{\text {ave }}$ is equal to $-8.15 \%$. As well, with increasing $\varphi$ the Nusselt number generally increases; the highest increase in $\mathrm{Nu}_{\text {ave }}$ equals to $13.8 \%$.

4. In $\mathrm{Ra}=10^{6}$, an increase in the Hartmann number reduces the average Nusselt number, and the highest decrease in $\mathrm{Nu}_{\text {ave }}$ is $-23.4 \%$. Furthermore, with increasing $\varphi$ the Nusselt number increases; the maximum increase in $\mathrm{Nu}_{\text {ave }}$ is equal to $11.2 \%$.

5. In $\mathrm{Ra}=10^{7}$ and $\mathrm{Ha}=0$, the flow field would be asymmetric and unsteady.

6. In $\mathrm{Ra}=10^{7}$, increasing Hartmann number reduces the average Nusselt number; the highest decrease in $\mathrm{Nu}_{\text {ave }}$ is equal to $-27.3 \%$. As well, the highest increase in $\mathrm{Nu}_{\text {ave }}$ with respect to $\varphi$ is $13.8 \%$.

7. An equation for the average Nusselt number as a function of mentioned parameters is presented.

Two dimensional numerical modelling and limited range of various parameters are the limitations of the presented study. Higher range of Rayleigh number and three dimensional numerical modelling can be recommended for future research. Transient behaviour of the flow regime at high Rayleigh number is another research area which can be recommended.

\section{Compliance with ethical standards}

Conflict of interest The authors declare that they have no known competing financial interests or personal relationships that could have appeared to influence the work reported in this paper.

Open Access This article is licensed under a Creative Commons Attribution 4.0 International License, which permits use, sharing, adaptation, distribution and reproduction in any medium or format, as long as you give appropriate credit to the original author(s) and the source, provide a link to the Creative Commons licence, and indicate if changes were made. The images or other third party material in this article are included in the article's Creative Commons licence, unless indicated otherwise in a credit line to the material. If material is not included in the article's Creative Commons licence and your intended use is not permitted by statutory regulation or exceeds the permitted use, you will need to obtain permission directly from the copyright holder. To view a copy of this licence, visit http://creativecommons .org/licenses/by/4.0/.

\section{Appendix}

The utilized mesh schematic has been shown in Fig. 19. It should be mentioned that the mesh size used in the numerical modelling is finer than which is shown in Fig. 19. But the fine mesh wasn't clear enough, so a coarse mesh has been shown.

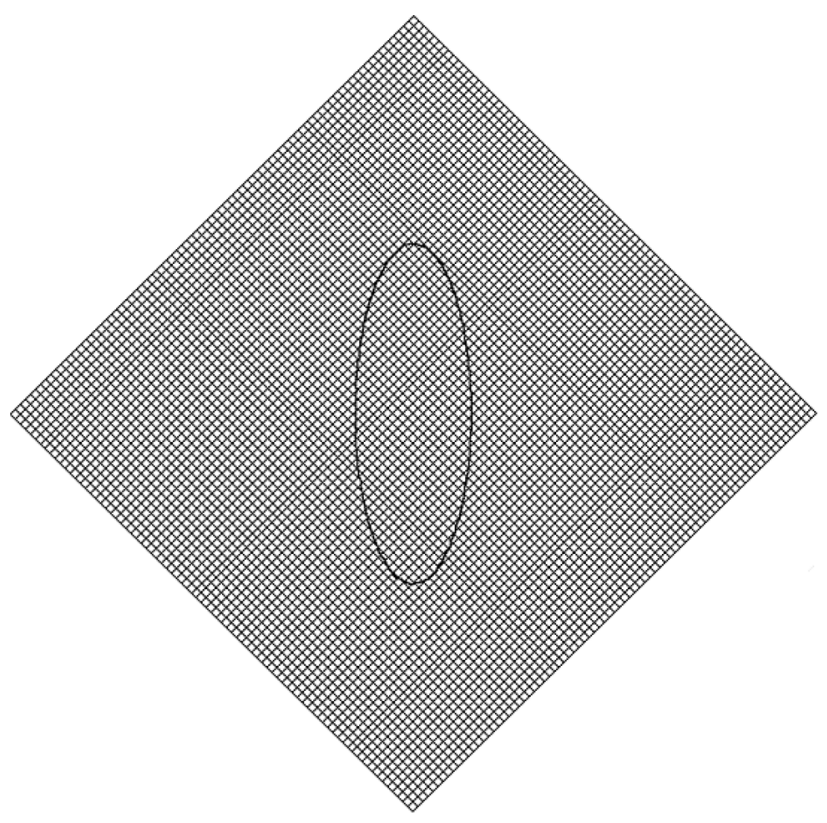

Fig. 19 A type of utilized mesh schematic

\section{References}

1. Vanaki SM, Ganesan P, Mohammed H (2016) Numerical study of convective heat transfer of nanofluids: a review. Renew Sustain Energy Rev 54:1212-1239

2. Öztop HF et al (2015) A brief review of natural convection in enclosures under localized heating with and without nanofluids. Int Commun Heat Mass Transf 60:37-44

3. Izadi S et al (2019) A comprehensive review on mixed convection of nanofluids in various shapes of enclosures. Powder Technol 343:880-907

4. Mahian O et al (2019) Recent advances in modeling and simulation of nanofluid flows-part i: fundamentals and theory. Phys Rep 790:1-48

5. Mahian O et al (2019) Recent advances in modeling and simulation of nanofluid flows - part ii: applications. Phys Rep 791:1-59

6. Devendiran DK, Amirtham VA (2016) A review on preparation, characterization, properties and applications of nanofluids. Renew Sustain Energy Rev 60:21-40

7. Angayarkanni S, Philip J (2015) Review on thermal properties of nanofluids: recent developments. Adv Coll Interface Sci 225:146-176

8. Azmi W et al (2016) The enhancement of effective thermal conductivity and effective dynamic viscosity of nanofluids-a review. Renew Sustain Energy Rev 53:1046-1058

9. Das PK (2017) A review based on the effect and mechanism of thermal conductivity of normal nanofluids and hybrid nanofluids. J Mol Liq 240:420-446 
10. Murshed SS, Estellé $P$ (2017) A state of the art review on viscosity of nanofluids. Renew Sustain Energy Rev 76:1134-1152

11. Sheikholeslami M, Rokni HB (2017) Simulation of nanofluid heat transfer in presence of magnetic field: a review. Int J Heat Mass Transf 115:1203-1233

12. Sarris I et al (2005) MHD natural convection in a laterally and volumetrically heated square cavity. Int J Heat Mass Transf 48(16):3443-3453

13. Oztop HF, Al-Salem K, Pop I (2011) MHD mixed convection in a lid-driven cavity with corner heater. Int J Heat Mass Transf 54(15-16):3494-3504

14. Kefayati GR (2013) Lattice Boltzmann simulation of natural convection in nanofluid-filled 2D long enclosures at presence of magnetic field. Theoret Comput Fluid Dyn 27(6):865-883

15. Hussein AK et al (2014) Lattice Boltzmann simulation of natural convection heat transfer in an open enclosure filled with $\mathrm{Cu}$-water nanofluid in a presence of magnetic field. Nucl Eng Des 268:10-17

16. Mahmoudi AH, Pop I, Shahi M (2012) Effect of magnetic field on natural convection in a triangular enclosure filled with nanofluid. Int J Therm Sci 59:126-140

17. Öztop HF et al (2012) MHD natural convection in an enclosure from two semi-circular heaters on the bottom wall. Int J Heat Mass Transf 55(7-8):1844-1854

18. Hussein AK et al (2016) Magneto-hydrodynamic natural convection in an inclined T-shaped enclosure for different nanofluids and subjected to a uniform heat source. Alex Eng J 55(3):2157-2169

19. Ghasemi $B$ (2013) Magnetohydrodynamic natural convection of nanofluids in U-shaped enclosures. Numer Heat Transf, Part A: Appl 63(6):473-487

20. Sheremet MA, Oztop H, Pop I (2016) MHD natural convection in an inclined wavy cavity with corner heater filled with a nanofluid. J Magn Magn Mater 416:37-47

21. Dogonchi A et al (2019) Numerical analysis of natural convection of $\mathrm{Cu}$-water nanofluid filling triangular cavity with semicircular bottom wall. JTherm Anal Calorim 135(6):3485-3497

22. Al-Rashed AA et al (2018) 3D magneto-convective heat transfer in CNT-nanofluid filled cavity under partially active magnetic field. Physica E 99:294-303

23. Pordanjani AH et al (2019) Effect of alumina nano-powder on the convection and the entropy generation of water inside an inclined square cavity subjected to a magnetic field: uniform and non-uniform temperature boundary conditions. Int J Mech Sci 152:99-117

24. Alsabery Al et al (2019) Two-phase nanofluid model and magnetic field effects on mixed convection in a lid-driven cavity containing heated triangular wall. Alex Eng J 59(1):129-148

25. Selimefendigil F, Öztop HF (2019) Corrugated conductive partition effects on MHD free convection of CNT-water nanofluid in a cavity. Int J Heat Mass Transf 129:265-277

26. Al-Rashed AA et al (2018) Three-dimensional investigation of the effects of external magnetic field inclination on laminar natural convection heat transfer in CNT-water nanofluid filled cavity. J Mol Liq 252:454-468

27. Sheikholeslami M, Gorji-Bandpy M, Ganji D (2013) Numerical investigation of MHD effects on Al2O3-water nanofluid flow and heat transfer in a semi-annulus enclosure using LBM. Energy 60:501-510

28. Ashorynejad HR, Mohamad AA, Sheikholeslami M (2013) Magnetic field effects on natural convection flow of a nanofluid in a horizontal cylindrical annulus using Lattice Boltzmann method. Int J Therm Sci 64:240-250

29. Selimefendigil F, Öztop HF (2014) MHD mixed convection of nanofluid filled partially heated triangular enclosure with a rotating adiabatic cylinder. J Taiwan Inst Chem Eng 45(5):2150-2162

30. Selimefendigil F, Öztop HF (2015) Natural convection and entropy generation of nanofluid filled cavity having different shaped obstacles under the influence of magnetic field and internal heat generation. J Taiwan Inst Chem Eng 56:42-56

31. Hassanpour A, Ranjbar A, Sheikholeslami M (2018) Numerical study for forced MHD convection heat transfer of a nanofluid in a square cavity with a cylinder of constant heat flux. Eur Phys J Plus 133(2):66

32. Kefayati GR, Tang H (2018) MHD thermosolutal natural convection and entropy generation of Carreau fluid in a heated enclosure with two inner circular cold cylinders, using LBM. Int J Heat Mass Transf 126:508-530

33. Barnoon P et al (2019) MHD mixed convection and entropy generation in a lid-driven cavity with rotating cylinders filled by a nanofluid using two phase mixture model. J Magn Magn Mater 483:224-248

34. Garmroodi MD, Ahmadpour A, Talati F (2019) MHD mixed convection of nanofluids in the presence of multiple rotating cylinders in different configurations: a two-phase numerical study. Int J Mech Sci 150:247-264

35. Alnaqi AA et al (2019) Effects of magnetic field on the convective heat transfer rate and entropy generation of a nanofluid in an inclined square cavity equipped with a conductor fin: considering the radiation effect. Int J Heat Mass Transf 133:256-267

36. Alkanhal TA et al (2019) Simulation of convection heat transfer of magnetic nanoparticles including entropy generation using CVFEM. Int J Heat Mass Transf 136:146-156

37. Tayebi T, Chamkha AJ (2020) Entropy generation analysis due to MHD natural convection flow in a cavity occupied with hybrid nanofluid and equipped with a conducting hollow cylinder. $J$ Therm Anal Calorim 139(3):2165-2179

38. Tayebi T, Chamkha AJ (2020) Magnetohydrodynamic natural convection heat transfer of hybrid nanofluid in a square enclosure in the presence of a wavy circular conductive cylinder. J Therm Sci Eng Appl 12(3):031009. https://doi.org/10.1115/1.4044857

39. Vijaybabu T, Dhinakaran S (2019) MHD Natural convection around a permeable triangular cylinder inside a square enclosure filled with $\mathrm{Al} 2 \mathrm{O} 3-\mathrm{H} 2 \mathrm{O}$ nanofluid: an LBM study. Int J Mech Sci 153:500-516

40. Hosseinjani AA, Nikfar M (2020) Numerical analysis of unsteady natural convection from two heated cylinders inside a rhombus enclosure filled with Cu-water nanofluid. Int Commun Heat Mass Transf 113:104510

41. Ashrafizadeh A, Hosseinjani AA (2017) A phenomenological study on the convection heat transfer around two enclosed rotating cylinders via an immersed boundary method. Int J Heat Mass Transf 107:667-685

42. Hosseinjani AA, Ashrafizadeh A (2017) An extended iterative direct-forcing immersed boundary method in thermo-fluid problems with dirichlet or neumann boundary conditions. J Cent South Univ 24(1):137-154

43. Corcione $M$ (2011) Empirical correlating equations for predicting the effective thermal conductivity and dynamic viscosity of nanofluids. Energy Convers Manage 52(1):789-793

44. Khanafer K, Vafai K, Lightstone M (2003) Buoyancy-driven heat transfer enhancement in a two-dimensional enclosure utilizing nanofluids. Int J Heat Mass Transf 46(19):3639-3653

45. Peskin CS (2002) The immersed boundary method. Acta Numerica 11:479-517

46. Ji C, Munjiza A, Williams J (2012) A novel iterative direct-forcing immersed boundary method and its finite volume applications. $J$ Comput Phys 231(4):1797-1821

47. Sheikholeslami M, Gorji-Bandpy M, Ganji D (2014) Lattice Boltzmann method for MHD natural convection heat transfer using nanofluid. Powder Technol 254:82-93

Publisher's Note Springer Nature remains neutral with regard to jurisdictional claims in published maps and institutional affiliations. 